\title{
Protein citrullination was introduced into animals by horizontal gene transfer from cyanobacteria
}

Thomas F. M. Cummings ${ }^{1 *}$, Kevin Gori ${ }^{2}$, Luis Sanchez-Pulido ${ }^{1}$, Gabriel Gavrilidis ${ }^{1 \dagger}$, David Moi ${ }^{3,4}$, Abigail R. Wilson ${ }^{1}$, Elizabeth Murchison ${ }^{2}$, Christophe Dessimoz $^{3,4,5}$, Chris P. Ponting ${ }^{1}$ and Maria A. Christophorou ${ }^{1,6^{* *}}$.

1 MRC Human Genetics Unit, The Institute of Genetics and Molecular Medicine, University of Edinburgh, Western General Hospital, Crewe Road, Edinburgh EH4 2XU, United Kingdom

${ }^{2}$ Transmissible Cancer Group, Department of Veterinary Medicine, Madingley Road, Cambridge CB3 OES, United Kingdom

${ }^{3}$ Department of Computational Biology, and Center for Integrative Genomics, University of Lausanne, Genopode, 1015 Lausanne, Switzerland

${ }^{4}$ Swiss Institute of Bioinformatics, 1015 Lausanne, Switzerland

${ }^{5}$ Department of Genetics Evolution and Environment, and Department of Computer Science, University College London, Darwin Building, Gower Street, London, WC1E 6BT

${ }^{6}$ The Babraham Institute, Cambridge, CB22 3AT, United Kingdom

${ }^{\dagger}$ Current address: Department of Biochemistry, University of Oxford, South Parks Road, Oxford OX1 3QU, United Kingdom

* Correspondence and requests for materials: maria.christophorou@babraham.ac.uk; tfmcummings@gmail.com

** Lead contact: maria.christophorou@babraham.ac.uk

\begin{abstract}
Protein post-translational modifications (PTMs) add an enormous amount of sophistication to biological systems but their origins are largely unexplored. Citrullination, a key regulatory mechanism in human physiology and pathophysiology, is particularly enigmatic in an evolutionary context. The citrullinating enzymes peptidylarginine deiminases (PADIs) are ubiquitous across vertebrates but absent from yeast, worms and flies. Here, we map the surprising evolutionary trajectory of PADls into the animal lineage. We present strong phylogenetic support for a clade encompassing animal and cyanobacterial PADIs that excludes fungal and other bacterial homologues. The animal and cyanobacterial PADls share unique, functionally relevant synapomorphies that are absent from all other homologues. Molecular clock calculations and sequence divergence analyses using the fossil record estimate the last common ancestor of the cyanobacterial and animal PADIs to be approximately 1 billion years old, far younger than the 3.35-4.52 billion years known to separate bacterial and eukaryotic lineages. Under an assumption of vertical descent, PADI sequence change is anachronistically slow during this evolutionary time frame, even when compared to mitochondrial proteins, products of likely endosymbiont gene transfer and some of the most highly conserved proteins in life. The consilience of evidence indicates that PADIs were introduced from cyanobacteria into animals by horizontal gene transfer (HGT). The ancestral cyanobacterial protein is enzymatically active and can citrullinate eukaryotic proteins, suggesting that the PADI HGT event introduced a new catalytic capability into the regulatory repertoire of animals. This study reveals the unusual evolution of a pleiotropic protein modification with clear relevance in human physiology and disease.
\end{abstract}




\section{Introduction}

Post-translational modifications (PTMs) allow for temporal and spatial control of protein function in response to cellular and environmental signals and comprise an integral part of cellular and organismal life. The development of ever more sensitive and quantitative analytical methods has made possible the identification of PTMs within cells and has enhanced our understanding of the molecular and cellular functions they regulate. This has led to renewed interest in studying previously known, as well as newly identified modifications. Although PTMs have been classically studied in eukaryotic organisms, an increasing number of them are also discovered in bacteria ${ }^{1,2}$. However, very few of the $>200$ currently known PTMs have been studied in an evolutionary context. Some PTMs, such as phosphorylation, acetylation and glycosylation are ubiquitous across all domains of life suggesting that the enzymes that catalyse them existed in the Last Universal Common Ancestor $(\text { LUCA })^{3}$. In other cases, such as protein ubiquitylation, evolutionary analyses show that the catalyzing enzymes only appear in the last eukaryotic common ancestor, although domains analogous to E1 enzymes can also be found in bacteria ${ }^{1,4}$. Understanding the processes and adaptive constrains that have shaped the evolution of protein modifying enzymes can reveal which aspects are likely to be functionally important and may inform our understanding of their organismal roles.

Citrullination is the post-translational conversion of a protein arginine residue to the non-coded amino acid citrulline and is catalysed by PADIs enzymes in a calcium-dependent manner. Although citrullination involves a small mass change of only $0.98 \mathrm{Da}$, the removal of a positive charge from the arginine side chain can lead to profound biochemical changes and is known to alter protein structure, sub-cellular localisation and affinity to other proteins and nucleic acids ${ }^{5-11}$. Via these alterations PADIs regulate fundamental physiological and cellular processes. The bestestablished role of citrullination is in innate immunity, through mediating the release of neutrophil extracellular traps (NETs) ${ }^{12}$ but a plethora of studies have shown that PADIs also regulate gene expression, chromatin compaction, nerve myelination, skin homeostasis and the establishment of ground state pluripotency ${ }^{8,13,14}$. Notably, deregulation of PADIs is strongly implicated in the aetiology of a host of pathologies including autoimmunity (rheumatoid arthritis, ulcerative colitis, psoriasis and type I diabetes), neurodegeneration (multiple sclerosis, Alzheimer's and prion diseases) and metastatic cancer ${ }^{13,15-18}$. In the case of rheumatoid arthritis, autoantibodies against citrullinated endogenous proteins (Anti-Citrullinated Protein Antibodies, ACPAs) serve as diagnostic and prognostic markers as they precede the onset of symptoms by several years and correlate with disease severity and response to treatment ${ }^{19}$. Experimental in vivo models of PADI over-expression show that deregulation of citrullination drives the development of multiple sclerosis and cancer $^{16,20}$, while genetic ablation or chemical inhibition of different PADI family members has been shown to mitigate against some the pathologies mentioned above ${ }^{16,21-23}$. Although the underlying mechanism in the above cases is aberrantly high citrullination, loss of PADI activity is also deleterious and has been shown to compromise neurodevelopment, fertility and embryo development ${ }^{8,14,24}$. PADIs have therefore emerged as important therapeutic targets ${ }^{25}$ and this has motivated the study of their exquisite regulation by calcium and the physiologically relevant mechanisms of their activation ${ }^{26-28}$.

In an evolutionary context, $P A D / s$ are puzzling. Orthologues of the human $P A D / s$ are ubiquitous in bony fish, birds, reptiles, amphibians and mammals, but are unexpectedly missing from many eukaryotes including plants, yeast, worms and 
insects. Indeed, their absence from these genetic model organisms has arguably impeded progress towards the understanding of their functions and citrullination has remained a rather obscure PTM for a long time. The PADI gene is widely thought to have appeared first in the last common ancestor of teleosteans and mammals ${ }^{13,29,30}$, with duplications in subsequent lineages resulting in five mammalian paralogues. Therefore, citrullination seemingly defies the perception that PTMs are of ancient origin.

Studying the evolutionary origin of PTM-catalyzing enzymes using protein sequence is particularly challenging as sequence similarity becomes less reliable with evolutionary distance, however structural homology can point to the evolutionary origins of proteins ${ }^{3,31}$. We therefore considered homology of PADI structural domains. Mammalian PADIs consist of three structural domains, the N-terminal (PAD_N, Pfam annotation: PF08526), middle (PAD_M, Pfam annotation: PF08527) and catalytic Cterminal domains (PAD_C, Pfam annotation: PF03068). Mammalian genomes encode two distant homologues of the PAD_C domain: $N(G), N(G)$-dimethylarginine dimethylaminohydrolase [DDAH] and Glycine amidinotransferase [AGAT]. Both, however, are divergent in sequence and lack PAD_N and PAD_M domains. Two other citrullinating enzymes are known among some bacteria and early-branching eukaryotes: pPAD, an extended agmatine deiminase found in Porphyromonas gingivalis and giardiaADI, an extended form of the free $L$-arginine deiminase $g A D I$, found in the human parasite Giardia Lamblia ${ }^{32,33}$. These enzymes also lack PAD_N and PAD_M domains, are highly divergent in sequence and have different substrate specificities.

Although PADI proteins are widely considered to be specific to vertebrates, their crystal structures ${ }^{28,34}$ hint at a possibly more ancient origin as they reveal that the catalytic (PAD_C) domain adopts the same pentein fold as a variety of other widely distributed proteins that otherwise show little similarity in terms of amino acid conservation $^{35,36}$ (Figure S1). These proteins include a broad family of guanidinogroup (the functional group of the side chain of arginine and agmatine) modifying enzymes that possess hydrolase, dihydrolase and amidinotransferase catalytic activity, such as bacterial and early eukaryotic agmatine deiminases. The penteinfold containing enzymes share a broad catalytic core of a Cys, His and two polar guanidine binding residues - Asp or $\mathrm{Glu}^{36}$. The presence of this ancient fold and catalytic triad within PAD_C suggests that it was present early in cellular life.

A 2015 study by Crisp et al., identified possible PADI homologues in some bacterial species. Based on the finding that a possible homologue could be identified in prokaryotes but not in multiple Drosophila and Caenorhabditis species, the authors included PADIs among a list of 145 genes proposed to have been transferred into the genome of a vertebrate ancestor of extant mammals by horizontal gene transfer (HGT, also known as lateral gene transfer) ${ }^{37}$. HGT is the non-heritable transmission of genetic material from one organism to another, often via a virus or mobile genetic element and involving endosymbiotic or commensal relationships between donor and recipient ${ }^{38,39}$. HGT is widespread among prokaryotes and is recognised as a mechanism that shapes the evolution and adaptive potential of bacteria, for example in the acquisition of antibiotic resistance ${ }^{40,41}$. Although many cases of horizontal transfer have been reported between bacteria and unicellular eukaryotes, fewer bacteria-to-animal HGT events have been studied to date ${ }^{38,42,43}$. The majority of cases involve transfer into an invertebrate host, such as an insect or worm ${ }^{44-48}$, while HGT into animals with specialised germline cells is thought to be very rare ${ }^{49}$. Furthermore, these few accounts of bacteria-to-animal HGT have been the topic of intense debate $e^{37,50-54}$. Claims of HGT should therefore be considered on a case-by- 
case basis and tested against the alternative hypothesis of widespread gene loss ${ }^{51}$. In light of the absence of PADI homologues in most invertebrate animals, PADI evolution requires detailed consideration.

\section{Results}

\section{Comprehensive Identification of PADI homologues}

In order to understand the distribution and evolution of citrullination we sought to identify all $P A D I$ homologues from across life. We started by collecting orthologous $P A D / s$ using the EggNOG database, employing an unsupervised clustering algorithm of all proteins contained in 2031 genomes across cellular life ${ }^{55}$. To expand on this list, we used HMMER searches to identify all sequences in current sequence databases that contain a PAD_C domain, as defined by having significant sequence similarity (E-value $\left.<1 \times 10^{-3}\right)$, and assessed these for the presence of critical substrate-binding and calcium-binding residues annotated to human PADIs ${ }^{28}$. This was supplemented by additional iterative jackhmmer searches and Position-Specific Iterated BLAST (PSI-BLAST) searches as well as tblastn searches of genomic databases.

The taxonomic distribution of $P A D / s$ and proportion of species that harbour a PADI orthologue are presented in Table 1. PADIs are not ubiquitous across the metazoa, but are found in all major branches of the vertebrates (jawless fish, sharks and rays, bony fish, amphibians, reptiles, birds and mammals). Out of all species whose genomes have been sequenced to date, the earliest diverging invertebrate animals with a PADI gene are Priapulus caudatus (an ecdysozoan), Saccoglossus kowalevskii (a hemichordate), and Branchiostoma belcheri (a cephalochordate). Surprisingly, we identified a large number of PADI sequences with conservation of substrate and calcium-binding residues in bacteria and fungi. PADIs are also not ubiquitous across bacteria, and are most prevalent within cyanobacteria. None of the known plants or animals diverging before opisthokonts have a detectable PADI homologue. Our searches also returned two outliers, one in archaea and one in viruses. However, upon closer inspection, both hits were determined to be due to misattribution (Figures S2, S3; see also Methods) and were therefore not included in further analyses. This taxonomic distribution could suggest an evolutionary model in which $P A D I$ genes were lost independently in many separate lineages. In this scenario, gene loss occurred in all early-branching lineages leading to at least 306 non-opisthokont eukaryotes and in other lineages, for example those leading to Drosophila and Caenorhabditis.

To test whether bacterial and fungal PADls are related to PPAD and gADI enzymes, we conducted an unrooted maximum likelihood phylogenetic analysis using putative PADI homologues and sequences with significant HMMER similarity to pPAD and gADI sequences (Figure S4). Bacterial, fungal and animal PADIs form a single monophyletic outgroup that excludes both PPAD and gADI enzyme types, showing that each of the three types of protein is phyletically distinct. The pPAD and gADI type proteins can therefore be excluded from further consideration of the evolutionary origin of animal PADIs. 


\section{A strongly supported phylogenetic clade contains cyanobacterial and animal but not fungal PADIs}

We next performed detailed phylogenetic analyses. All bacterial PADI sequences within the Pathosystems Resource Integration Center (PATRIC) database were aligned with a subsampled set of PADI sequences from animals and fungi selected to maximise both protein sequence diversity and lineage representation. A maximum likelihood approach with the best fitting evolutionary rate model was used to produce an initial phylogenetic tree. Very strong bootstrap support $(>95 \%)$ was obtained for a clade restricted to certain cyanobacterial and animal PADIs that excludes a fully supported outgroup clade containing fungal, actinobacterial and proteobacterial sequences (Figure S5). Full node support placed fungal PADIs in a clade with actinobacterial sequences to the exclusion of animal PADI sequences. This tree topology, whereby animal sequences have closer affinity to those in cyanobacteria than they have to other eukaryotic (fungal) sequences is surprising because it is inconsistent with the known species tree.

Phylogenetic methods, in particular phylogenetic trees built on single genes, are not infallible. It is therefore possible that the observed topology represents the failure of phylogenetic inference in the case of this individual gene, such that an artefact (e.g. model misspecification) might explain the affinity of the separate eukaryotic PADIs to different bacterial PADI types. This might be indicated for example by a changing tree topology under different models of rate variation. A common approach for an initial maximum likelihood analysis uses a best-fitting (maximum likelihood) fixed rate matrix of amino acid substitutions to produce the tree ${ }^{56-58}$. This can be confounded if there is evolutionary rate variation over different parts of the tree or deviation from typical protein substitution rates. In particular, attention has been drawn previously to heterotachous evolution, where the evolutionary substitution rate of a given site may change over time ${ }^{59}$. Heterotachy might be particularly plausible in the case of $P A D I$, given the very divergent species represented in the analysis (animal, fungal, cyanobacterial, actinobacterial).

To validate the tree topology, and informed by the large tree, bacterial PADI sequences were subsampled to cover a broad representation of orthologous sequences for more computationally expensive phylogenetic analyses (selected to maximise diversity of bacterial $P A D I$ sequences as determined by varied representation of bitscore similarity to the animal PADI sequences). Three approaches were used to address possible heterotachous effects: a Bayesian approach that samples over different fixed empirical rate matrices ${ }^{60}$; a maximum likelihood approach using a mixture model of 20 different fixed amino acid rate matrices $(\mathrm{C} 20)^{61}$; and a Bayesian approach that allows for infinite mixture model categories sampled from the alignment by making use of a Dirichlet process prior $(\text { CAT-GTR })^{62}$. These methods have been shown to be more robust to long-branch artefacts, and to saturated sequence artefacts more broadly, due to the more sophisticated treatment of across-site rate heterogeneity ${ }^{63-66}$.

All of the above analyses recovered a single topology that supports a clade of cyanobacterial and animal sequences to the exclusion of a clade of fungal and actinobacterial sequences (Figure 1a). Posterior probabilities or bootstrap values for this topology were high, approaching $100 \%$ for each of the four diverse methods (Figure 1b). The analysis was repeated using additional bootstrap algorithms, including the full non-parametric bootstrap, obtaining full support ${ }^{67,68}$. Topology constraint tests rejected a number of randomly generated trees to rule out possible 
specific biases in bootstrap resampling. Lastly, we generated a constrained tree for the expected model where eukaryotic PADIs are restricted to a monophyletic group. These alternative trees and constraint tree were all significantly rejected $(p<0.0001)$ by multiple statistical tests including the $\mathrm{AU}$-test ${ }^{69-71}$.

\section{Cyanobacterial and animal PADIs share unique synapomorphies}

Although unlikely, some degree of stochastic evolution may potentially result in artefactual affinity of cyanobacterial PADIs with animal PADIs in the single gene tree. We therefore sought to identify features of the protein sequence that may independently validate the phylogenetic topology. A synapomorphy is any characteristic that is shared among multiple taxa but not with their ancestors. We identified three synapomorphies that are shared between animal and cyanobacterial PADI proteins but not with any actinobacterial or fungal PADIs (Figure 2).

Firstly, we examined how the PADI protein domain architecture is distributed across orthologues using Pfam annotations, which are powered by HMMER searches $^{72}$. As mentioned above, all metazoan PADls possess the three PADI domains, PAD_N, PAD_M and PAD_C (Figure S1). The cyanobacterial PADIs closest to mammalian PADIs (from SPM and $N X$ cyanobacteria) appear to possess two Pfam-annotated domains: a PAD_M domain and a PAD_C domain, but not a PAD_N domain. By contrast, other bacterial and fungal PADIs are only annotated with the PAD_C domain. To identify domains that might have been overlooked by Pfam, we carried out more sensitive profile-to-profile HMM searches ${ }^{73,74}$ (Figure $\mathrm{S6a}$ ). We made a multiple sequence alignment firstly of cyanobacterial species contained in the monophyletic clade of metazoan sequences (Figure 1a, Clade Ai), and secondly of the remaining bacterial and fungal sequences (Figure 1a, sequences outside of Clade Aii). Regions corresponding to each of the PAD_N, PAD_M and PAD_C domains from human PADI2 were extracted and searched against a database of profiles of all domains contained in Pfam. This revealed that the bacterial and fungal sequences outside Clade Aii possess a divergent version of the PAD_M domain, but do not possess any PAD_N domain: the PAD_N region is completely absent from those fungal and bacterial orthologues, including cyanobacteria diverging earlier than $S P M / N X$. By contrast, the cyanobacterial homologues contained within Clade $\mathrm{Ai}$ (diverging after SPM and $N X$ clades) possess all three domains including a degenerate metazoan PAD_N cupredoxin type domain (PAD_N domain: $E$-value $<1 \times 10^{-7}$ ). We then identified the cyanobacterial sequence that is predicted to assume the PAD_N secondary structure using PsiPred and aligned this with animal PAD_N sequences. The predicted cyanobacterial PAD_N sequence aligns well with the human PAD_N domain, as determined experimentally using PADI2 crystal structure data ${ }^{28}$ (Figure 2a), confirming that the Clade Ai cyanobacterial PADIs possess a degenerate PAD_N domain.

Secondly, we analysed representative fungal, actinobacterial, cyanobacterial, and metazoan PADI sequences for the conservation of calcium-binding and active site residues (Figure 2b). The allosteric binding of up to six calcium ions allows formation of the PADI2 active site cleft and is an absolute requirement for catalytic activity $^{28}$. All catalytic residues and substrate binding residues are fully conserved among all PADI homologues (Figure $2 \mathrm{~b}$ ). In addition, calcium-binding sites 3 and 1 appear to be fully conserved, while calcium site 5 is also likely conserved. Ca6 is likely to be conserved functionally, as the substitution of D125 to N and E131 to D, which are present in both actinobacterial and fungal sequences, are expected to preserve ion binding. Intriguingly, however, calcium sites 2 and 4 appear to be exclusive to Clade Ai (late diverging cyanobacterial and metazoan) sequences. The 
fungal and actinobacterial sequences diverge from binding sites 2 and 4 to a different amino acid motif. Critically, only Clade Ai PADI sequences conserve the calcium switch residue D389 (residues: 369-389). In actinobacterial and fungal sequences this residue is substituted to Gly and therefore incompetent for metal coordination ${ }^{28}$ (Figure 2b). This indicates that the ordered, sequential calcium binding in the PAD_M domain, which is responsible for the allosteric communication between PAD_M and the catalytic PAD_C domain in human PADI ${ }^{28}$ is likely to be conserved only in Clade Ai PADIs. As a result, a potentially different mode of calcium regulation operates in the fungal and actinobacterial PADIs.

Additionally, we find that fungal and actinobacterial sequences share features that are not present in the Clade Ai PADIs. This includes a conserved region within calcium binding sites 3-5 that is absent from the metazoan and cyanobacterial sequences (Figure 2b: amino acids 155-180, where differences conserved between fungal and actinobacterial sequences are highlighted in yellow). Also of interest is a highly conserved 10 amino acid beta sheet that connects the PAD_M and PAD_C domains (Figure 2b: amino acids 292-302). This region is conserved closely in fungal and actinobacterial sequences, but to a different 10 amino acid sequence containing a distinctive triple histidine motif (Figure $2 \mathrm{~b}$ : amino acids 300-302).

The phylogenetic topology presented above is consistent whether built with or without the above synapomorphic sequence features and PAD_N domain (Figure $\mathrm{S6b})$. As these synapomorphic features occur at the level of the amino acid sequence and at the level of a whole protein domain (Figure 2c), they are robust to convergent evolution, to differences in rate variation across the tree and to saturated sequence artefacts ${ }^{75-78}$. These features therefore provide strong additional support of the phylogenetic topology presented in Figure 1. It is inconceivable that blocks of sequence of up to ten amino acids were derived convergently and independently in actinobacterial and fungal PADIs. Thus these sequence features indicate a common ancestry of actinobacterial and fungal PADIs that is distinct from the ancestry of cyanobacterial and metazoan PADIs.

\section{The PADI sequence evolution between cyanobacteria and animals is anachronistically slow}

The remarkably high similarity of Clade Ai cyanobacterial and animal PADIs prompted us to examine the rate of sequence change between them in more detail. We used a Bayesian phylogenetic approach to predict the divergence time between Ai Clade cyanobacterial and animal PADI sequences under a strict molecular clock model, using known fossil ages of metazoans as calibrations ${ }^{79-81}$. This prediction is expected to be approximately 3.35 billion years, in line with the age of the last common ancestor known to separate bacteria and eukarya ${ }^{82}$ (or greater, if evolutionary rates deviated in either lineage). Instead, our analysis yielded a far younger estimate of approximately 1 billion years (Figure 3a) for the age of their last common ancestor. Estimating divergence times using several relaxed clock models (UCLN, UCED, random local clocks ${ }^{81}$ ) increased the uncertainty in the estimate but, in all three cases, estimated an even more recent mean divergence time (Figure 3b). Under all approaches, the divergence times were not congruent with the geologicallydefined divergence $\left(p<10^{-8}\right)$ (Figure 3b). These divergence time estimates are therefore inconsistent with vertical descent of metazoan PADIs and are instead consistent with a horizontal acquisition event, which is more recent than the acquisition of the mitochondrion by eukarya. The divergence times predicted by 
these clock models are approximately dated at the time of divergence of the last common ancestor of PADI-harbouring metazoa.

Since the divergence of the PADI sequence between cyanobacteria and metazoa was unexpectedly low, we sought to understand it in the context of the genetic divergence between the species that bridge the closest PADI homologues. We therefore analysed a large number of the most conserved proteins in life to approximate a mean minimum extent of accumulated genetic divergence occurring between Cyanothece sp. 8801 and Branchiostoma belcheri and compared this to the divergence of the PADI sequence between these two species (Figure S7). The distribution of calculated accumulated genetic divergence for 26 highly conserved proteins (ribosomal proteins, essential metabolic enzymes and chaperones) did not deviate significantly from a normal distribution (Figure S7). As a positive control for the hypothesised horizontal trajectory, we also analysed 19 proteins of likely endosymbiont gene transfer (EGT) origin and 10 proteins encoded in the mitochondrial genomes. Since mitochondrial and EGT-derived proteins were acquired more recently than the LUCA, the mean of the total accumulated sequence change for each of these proteins is expected to be much lower than that for vertically transferred genes (Figure S7). We therefore reasoned that they may mimic more closely the extent of accumulated genetic divergence that would be expected for an anciently horizontally transferred gene acquired more recently than the mitochondrion (as is hypothesised for the PADI gene). As expected, EGT and mitochondrially encoded proteins have an average accumulated genetic divergence that is significantly lower than that of vertically acquired proteins (Figure 3c). Specifically, the total accumulated genetic divergence for PADI sequences falls 6 standard deviations below that calculated for vertically transferred protein sequences, as assessed over the same timescale (Figure $3 c$ ). PADIs show less sequence change than all proteins individually analysed over this timescale and less even than ribosomal RNA. Indeed, they fall 2 standard deviations below the mean of EGT candidate genes or the mean of genes derived from the mitochondrial genome (Figure 3c). In a model of vertical descent, PADls would therefore be under greater constraint than any other known sequence ${ }^{83}$.

\section{The cyanobacterial PADI protein is catalytically active}

Considering the high degree of similarity between Clade Ai cyanobacterial and metazoan PADIs, including all necessary catalytic residues and calcium binding residues, we hypothesised that the ancestral cyanobacterial enzyme is likely to be catalytically active and calcium dependent. To test this, we prepared a recombinant version of the three-domain PADI from Cyanothece sp. 8801 (here referred to as "cyanoPADI") and assayed its catalytic activity alongside human PADI4. Analogously to the human enzyme, cyanoPADI can citrullinate multiple proteins in mouse cell lysates (Fig. 4a). In addition, cyanoPADI shows absolute dependence on calcium for activity. This demonstrates that the calcium-dependent regulation found in mammalian PADIs is also a feature of the ancestral cyanobacterial protein and suggests that the conserved calcium-binding sites, which were used in the evolutionary analysis as signifiers of synapomorphy, are functional (Fig. $2 \mathrm{~b}$ and Fig. 4). Remarkably, and despite the absence of histones from bacteria, cyanoPADI catalyses citrullination of histone $\mathrm{H} 3$ (Fig. 4b), which is a known target of mammalian PADI4. The enzyme is additionally active at a physiologically relevant temperature for cyanobacteria (Fig. 4b). Thus cyanoPADI is a bona fide calcium-dependent peptidylarginine deiminase with sufficient similarity or promiscuity to catalyse citrullination of mammalian substrates. 


\section{Discussion}

It has been hypothesised that very few protein modification types existed in the LUCA and these have been diversified to give rise to the $>200$ PTMs known today $^{3}$. We sought to map the evolutionary origin of citrullination, which is implicated in the regulation of an ever-increasing number of physiological and pathological processes. Our analyses of PADI homologues across life reveal the existence of two clearly discernible types of PADI homologues: one containing three structural domains and sharing functionally relevant sequence features and one containing two structural domains and divergent sequence features. The taxonomic distribution of these two types of homologues is highly unusual, in that three-domain PADIs are present in animal and late-diverging cyanobacteria, while two-domain PADIs are present in fungi and all other bacteria (Figures 1,2, S6). This evidence can be reconciled with vertical evolutionary descent if the LUCA harboured two paralogous $P A D I$ genes which underwent widespread and mutually exclusive losses throughout evolution: firstly, the three-domain $P A D I$ present in late-diverging cyanobacteria and metazoa was lost from lineages leading to every other species in life; and secondly, the two-domain PADI present in fungi, actinobacteria and proteobacteria must be separately accounted for in independent gene losses in lineages leading to all other species. In lineages that harbour no PADI, the two paralogues must have been lost independently.

This highly unparsimonious scenario would be supported if rates of PADI sequence evolution across a species phylogeny were consistent with respect to geologically defined timings and with genes well known to have been inherited vertically from the LUCA. Our analyses of sequence divergence provide evidence to the contrary. In absolute terms, the similarity of cyanobacterial and branchiostomal PADIs to human PADIs is almost identical: $70.20 \%$ vs $70.90 \%$ respectively by pairwise amino acid similarity. However, a much greater amount of time has elapsed since the cyanobacterial and human genes have shared a last common ancestor than the genes from the other species pair (branchiostoma and humans). Even under assumptions of heterotachy, where rates of evolution may differ between different lineages, a minimal amount of nearly neutral genetic divergence nonetheless accumulates over evolutionary timescales in all lineages ${ }^{83}$. Under the assumption of vertical descent, the observed PADI sequence changes are anachronistically low even compared to the most highly conserved genomic sequences in life, including EGT candidates and mitochondrial genes.

The explanation for the observation of such little sequence change is more mundane under the assumption of horizontal transfer. A HGT event from latediverging SPM/NX clade cyanobacteria to a last common ancestor within the animal lineage, although ancient, would have occurred much more recently than the LUCA and also likely more recently than the mitochondrion. HGT can therefore fully account for the phylogenetic distribution as well as the slow rates of evolution observed. The two lines of evidence are complementary and independent. The timing of transfer (neoproterozoic: 1000-542MYA) is consistent with the presence of marine nitrogen fixing cyanobacteria with specialised arginine catabolic pathways ${ }^{84}$, and with the emergence of metazoa in the cyanobacterial habitat ${ }^{85-87}$. A second HGT event, from actinobacterial species that are known to be fungal pathogens, most parsimoniously explains the existence of the two-domain fungal PADI (Clade $\mathrm{Bi}$, Figures 1, S4 and Figure 2). This is consistent with the absence of a PADI gene either in eukaryotic species diverging before opisthokonts or in early diverging fungi such as yeast.

Closer examination of $P A D I$ phylogeny in bacteria provides additional support for HGT and confirms the directionality of horizontal transfer (Figure S8 and S9). Firstly, strong support is found for bacterial PADIs that form an outgroup to both the two-domain and three-domain PADI sequences (Figure S5 and S9). These bacterial outgroup sequences suggest that PADIs were not horizontally acquired by bacteria. 
Secondly, the fact that the metazoan-type three-domain PADI only emerges in the late-diverging SPM and $N X$ clades of cyanobacteria, and the cyanobacterial PADI phylogeny mirrors the expected species tree ${ }^{88}$ (Figure S8), indicates that the threedomain PADI did not exist in the LUCA. The existence of cyanobacterial outgroup sequences, with a discernable origin within bacterial evolution, specifically implies the direction of HGT of the three-domain PADI was from cyanobacteria into metazoa and not in reverse (Figure S8).

All but one metazoan PADI sequence identified by our comprehensive searches in genomic and proteomic databases were found in deuterostomes - the exception being found in the Priapulus caudatus genome, a protostome. This suggests that the HGT took place either at the root of the deuterostomes, or possibly at the root of bilateria. Note that this part of the tree of life remains poorly resolved, with an extremely short branch between the bilaterian common ancestor and the deuterostomes ${ }^{89}$.

Biochemical analyses of the ancestral three-domain PADI (cyanoPADI) show that it is competent for catalysis (Figure 4), while a recent study has identified catalytically active PADI homologues in the thermotolerant fungi Emericella dentata and Aspergillus nidulans ${ }^{90}$. The discovery of catalytically active PADI orthologues in bacteria and fungi offers fertile ground for investigation of the roles of citrullination in these organisms.

Our finding that the cyanoPADI can citrullinate mammalian substrates (Figure 4 ) indicates that a novel catalytic capability was added to the regulatory repertoire of metazoan cells by HGT. The newly acquired regulatory function is likely to have enhanced biochemical diversity in animals. Fish genomes contain a single PADI gene, but duplications resulted in five tandem repeated paralogues in mammalian genomes ${ }^{91}$ (Figure S9). The fact that these duplicated genes were retained across many animal genomes suggests that they were unlikely to be functionally redundant. In the course of vertebrate evolution, citrullination was thus expanded in scope and adapted to a variety of cellular contexts, ranging from neutrophil extracellular trap release to stem cell potency, and from oligodendrocytes to bone marrow and keratinocytes ${ }^{30}$. The emerging physiological roles of the vertebrate PADIs, such as in the regulation of pluripotency and embryonic development ${ }^{8,24,92,93}$, or the newly described role of the fish PADI in tissue regeneration ${ }^{94}$, point to possible selective advantages conferred to metazoans by PADIs and offer a possible explanation for the fact that PADIs were retained so widely ${ }^{95}$. In a similar vein, it is interesting to consider our findings in light of the proposal that genes with a role antimicrobial defence are amenable to co-option by eukaryotic innate immune systems ${ }^{46}$. The extent to which the molecular mechanisms that regulate the human PADIs were also conserved from cyanobacteria or were newly co-opted in vertebrates remains an intriguing open question.

It is notable that no citrullination-reversing enzyme has been identified in any species to date. The evolutionary analysis of PADIs presented here adds extra complexity as to whether the reverse catalytic process might have also arisen or been propagated. It has been postulated that "toolkits" of PTM writer, eraser and reader enzymes may have evolved in a coordinated fashion and this has been studied formally in the context of protein phosphorylation ${ }^{96}$. In this context, the investigation into potential reverse catalysis for citrullination should be extended to include bacterial and fungal enzymes.

A related consideration is prompted by the known role of PADls in autoimmunity. It has been proposed that the exogenous citrullinating activity of pPAD at sites of periodontal infection is an initiating event in the development of RA, by predisposing individuals with prior periodontal infection to the development of $\mathrm{ACPAs}^{97}$. It is therefore of note that PPAD and gADI genes are more widespread 
than previously thought (Figure S4) and that the PADIs described in this paper can be found in a number of human pathogens and in Stachybotrys chlorohalonata (black mold). A re-evaluation of the initiating events responsible for citrullination-specific breaks in immune tolerance may therefore be warranted.

This work reveals the remarkable evolutionary trajectory of the PADI family of human genes and uncovers the origin of a pleiotropic regulatory protein modification. In combination, the pieces of evidence presented above comprise a compelling case of ancient horizontal transfer of a bacterial gene into animals.

\section{Author contributions}

T.F.M.C. and M.A.C. conceived the idea for the project and wrote the manuscript. T.F.M.C. and K.G. performed conservation, phylogenetic and time divergence analyses. T.F.M.C. performed domain architecture and structural analyses. L.S.P. performed structure-informed multiple sequence alignments. A.R.W. generated the vector for expression of recombinant cyanoPADI. G.G and T.F.M.C. performed protein expression and purification and carried out biochemical assays. C.D. and D.M advised on aspects of taxonomy and phylogeny. C.P.P. advised on aspects of structural and evolutionary biology. C.D and C.P.P. helped edit the manuscript.

\section{Acknowledgements}

This work was funded by a Wellcome Trust and Royal Society Sir Henry Dale Fellowship and a MRC/University of Edinburgh Chancellor's Fellowship to M.A.C. C.P.P. and L.S.-P. were funded by the Medical Research Council. D.M. and C.D. were funded by Swiss National Science Foundation Grant 183723. We thank M. Reijns for the gift of the pGEX-His plasmid, and G. Abrusán, G. Slodkowicz, M. Babu, N.D. Hastie and members of the Christophorou laboratory for critical discussions of the work. 


\section{References}

1. Koonin, E. V. The origin and early evolution of eukaryotes in the light of phylogenomics. Genome Biology (2010). doi:10.1186/gb-2010-11-5-209

2. Grangeasse, C., Stülke, J. \& Mijakovic, I. Regulatory potential of posttranslational modifications in bacteria. Frontiers in Microbiology (2015). doi:10.3389/fmicb.2015.00500

3. Beltrao, P., Bork, P., Krogan, N. J. \& Van Noort, V. Evolution and functional cross-talk of protein post-translational modifications. Molecular Systems Biology (2013). doi:10.1002/msb.201304521

4. Hochstrasser, M. Origin and function of ubiquitin-like proteins. Nature (2009). doi:10.1038/nature07958

5. Tanikawa, C. et al. Regulation of protein citrullination through p53/PADI4Network in DNA damage response. Cancer Res. 69, 8761-8769 (2009).

6. Guo, Q. \& Fast, W. Citrullination of Inhibitor of Growth 4 (ING4) by Peptidylarginine Deminase 4 (PAD4) disrupts the interaction between ING4 and p53. J. Biol. Chem. 286, 17069-17078 (2011).

7. Stadler, S. C. et al. Dysregulation of PAD4-mediated citrullination of nuclear GSK3 $\beta$ activates TGF- $\beta$ signaling and induces epithelialto-mesenchymal transition in breast cancer cells. Proc. Natl. Acad. Sci. U. S. A. (2013). doi:10.1073/pnas.1308362110

8. Christophorou, M. A. et al. Citrullination regulates pluripotency and histone $\mathrm{H} 1$ binding to chromatin. Nature 507, 104-108 (2014).

9. Snijders, A. P. et al. Arginine methylation and citrullination of splicing factor proline- and glutamine-rich (SFPQ/PSF) regulates its association with mRNA. RNA 21, 347-359 (2015).

10. Tanikawa, C. et al. Citrullination of RGG Motifs in FET Proteins by PAD4 Regulates Protein Aggregation and ALS Susceptibility. Cell Rep. 22, 14731483 (2018).

11. Sharma, P. et al. Arginine Citrullination at the C-Terminal Domain Controls RNA Polymerase II Transcription. Mol. Cell 73, 84-96.e7 (2019).

12. Wang, Y. et al. Histone hypercitrullination mediates chromatin decondensation and neutrophil extracellular trap formation. J. Cell Biol. (2009). doi:10.1083/jcb.200806072

13. Wang, S. \& Wang, Y. Peptidylarginine deiminases in citrullination, gene regulation, health and pathogenesis. Biochimica et Biophysica Acta - Gene Regulatory Mechanisms 1829, 1126-1135 (2013).

14. Falcão, A. M. et al. PAD2-Mediated Citrullination Contributes to Efficient Oligodendrocyte Differentiation and Myelination. Cell Rep. (2019). doi:10.1016/j.celrep.2019.03.108

15. Suzuki, A. et al. Functional haplotypes of PADI4, encoding citrullinating enzyme peptidylarginine deiminase 4 , are associated with rheumatoid arthritis. Nat. Genet. 34, 395-402 (2003).

16. Musse, A. A. et al. Peptidylarginine deiminase 2 (PAD2) expression in a 
transgenic mouse leads to specific central nervous system (CNS) myelin instability. Dis. Model. Mech. 1, 229-240 (2008).

17. Zhang, X. et al. Genome-Wide analysis reveals PADI4 cooperates with Elk-1 to activate C-Fos expression in breast cancer cells. PLoS Genet. 7, (2011).

18. Yuzhalin, A. E. et al. Colorectal cancer liver metastatic growth depends on PAD4-driven citrullination of the extracellular matrix. Nat. Commun. 9, (2018).

19. Willemze, A., Trouw, L. A., Toes, R. E. M. \& Huizinga, T. W. J. The influence of ACPA status and characteristics on the course of RA. Nature Reviews Rheumatology (2012). doi:10.1038/nrrheum.2011.204

20. McElwee, J. L. et al. PAD2 overexpression in transgenic mice promotes spontaneous skin neoplasia. Cancer Res. (2014). doi:10.1158/00085472.CAN-14-0749

21. Wei, L. et al. Novel inhibitors of protein arginine deiminase with potential activity in multiple sclerosis animal model. J. Med. Chem. (2013). doi:10.1021/jm301755q

22. Moscarello, M. A. et al. Inhibition of peptidyl-arginine deiminases reverses protein- hypercitrullination and disease in mouse models of multiple sclerosis. DMM Dis. Model. Mech. (2013). doi:10.1242/dmm.010520

23. Willis, V. C. et al. Protein arginine deiminase 4 inhibition is sufficient for the amelioration of collagen-induced arthritis. Clin. Exp. Immunol. (2017). doi:10.1111/cei.12932

24. $\mathrm{Xu}, \mathrm{Y}$. et al. Mutations in PADI6 Cause Female Infertility Characterized by Early Embryonic Arrest. Am. J. Hum. Genet. (2016). doi:10.1016/j.ajhg.2016.06.024

25. Lewis, H. D. \& Nacht, M. IPAD or PADi - 'tablets' with therapeutic disease potential? Current Opinion in Chemical Biology 33, 169-178 (2016).

26. Darrah, E. et al. Erosive rheumatoid arthritis is associated with antibodies that activate PAD4 by increasing calcium sensitivity. Sci. Transl. Med. 5, 186 ra65 (2013).

27. Neeli, I. \& Radic, M. Opposition between PKC isoforms regulates histone deimination and neutrophil extracellular chromatin release. Front. Immunol. 4, (2013).

28. Slade, D. J. et al. Protein arginine deiminase 2 binds calcium in an ordered fashion: Implications for inhibitor design. ACS Chem. Biol. 10, 1043-1053 (2015).

29. Balandraud, N. et al. A rigorous method for multigenic families' functional annotation: The peptidyl arginine deiminase (PADs) proteins family example. BMC Genomics (2005). doi:10.1186/1471-2164-6-153

30. Nicholas, A. P. \& Bhattacharya, S. K. Protein deimination in human health and disease. Protein Deimination in Human Health and Disease (2014). doi:10.1007/978-1-4614-8317-5

31. Scheeff, E. \& Bourne, P. Structural Evolution of the Protein Kinase-Like Superfamily. PLoS Comput. Biol. (2005). doi:10.1371/journal.pcbi.0010049.eor

32. Goulas, T. et al. Structure and mechanism of a bacterial host-protein citrullinating virulence factor, Porphyromonas gingivalis peptidylarginine 
deiminase. Sci. Rep. (2015). doi:10.1038/srep11969

33. Touz, M. C. et al. Arginine deiminase has multiple regulatory roles in the biology of Giardia lamblia. J. Cell Sci. (2008). doi:10.1242/jcs.026963

34. Arita, K. et al. Structural basis for Ca2+-induced activation of human PAD4. Nat. Struct. Mol. Biol. 11, 777-783 (2004).

35. Shirai, H., Blundell, T. L. \& Mizuguchi, K. A novel superfamily of enzymes that catalyze the modification of guanidino groups. Trends in Biochemical Sciences (2001). doi:10.1016/S0968-0004(01)01906-5

36. Linsky, T. \& Fast, W. Mechanistic similarity and diversity among the guanidinemodifying members of the pentein superfamily. Biochimica et Biophysica Acta - Proteins and Proteomics (2010). doi:10.1016/j.bbapap.2010.07.016

37. Crisp, A., Boschetti, C., Perry, M., Tunnacliffe, A. \& Micklem, G. Expression of multiple horizontally acquired genes is a hallmark of both vertebrate and invertebrate genomes. Genome Biol. (2015). doi:10.1186/s13059-015-0607-3

38. Boto, L. Horizontal gene transfer in the acquisition of novel traits by metazoans. Proceedings of the Royal Society B: Biological Sciences (2014). doi:10.1098/rspb.2013.2450

39. Soucy, S. M., Huang, J. \& Gogarten, J. P. Horizontal gene transfer: Building the web of life. Nature Reviews Genetics (2015). doi:10.1038/nrg3962

40. Ochman, H., Lawrence, J. G. \& Grolsman, E. A. Lateral gene transfer and the nature of bacterial innovation. Nature (2000). doi:10.1038/35012500

41. Koonin, E. V., Makarova, K. S. \& Aravind, L. Horizontal Gene Transfer in Prokaryotes: Quantification and Classification. Annu. Rev. Microbiol. (2001). doi:10.1146/annurev.micro.55.1.709

42. Keeling, P. J. \& Palmer, J. D. Horizontal gene transfer in eukaryotic evolution. Nature Reviews Genetics (2008). doi:10.1038/nrg2386

43. Dunning Hotopp, J. C. Horizontal gene transfer between bacteria and animals. Trends in Genetics (2011). doi:10.1016/j.tig.2011.01.005

44. Gladyshev, E. A., Meselson, M. \& Arkhipova, I. R. Massive horizontal gene transfer in bdelloid rotifers. Science (80-. ). (2008). doi:10.1126/science.1156407

45. Moran, N. A. \& Jarvik, T. Lateral transfer of genes from fungi underlies carotenoid production in aphids. Science (80-. ). (2010). doi:10.1126/science.1187113

46. Chou, S. et al. Transferred interbacterial antagonism genes augment eukaryotic innate immune function. Nature (2015). doi:10.1038/nature13965

47. Dunning Hotopp, J. C. Grafting or pruning in the animal tree: Lateral gene transfer and gene loss? BMC Genomics (2018). doi:10.1186/s12864-0184832-5

48. Lacroix, B. \& Citovsky, V. Transfer of DNA from bacteria to eukaryotes. MBio (2016). doi:10.1128/mBio.00863-16

49. Jensen, L., Grant, J. R., Laughinghouse, H. D. \& Katz, L. A. Assessing the effects of a sequestered germline on interdomain lateral gene transfer in Metazoa. Evolution (2016). doi:10.1111/evo.12935 
50. Stanhope, M. J. et al. Phylogenetic analyses do not support horizontal gene transfers from bacteria to vertebrates. Nature (2001). doi:10.1038/35082058

51. Salzberg, S. L. Horizontal gene transfer is not a hallmark of the human genome. Genome Biology (2017). doi:10.1186/s13059-017-1214-2

52. Martin, W. F. Too Much Eukaryote LGT. BioEssays (2017). doi:10.1002/bies.201700115

53. Leger, M. M., Eme, L., Stairs, C. W. \& Roger, A. J. Demystifying Eukaryote Lateral Gene Transfer. BioEssays (2018). doi:10.1002/bies.201700242

54. Husnik, F. \& McCutcheon, J. P. Functional horizontal gene transfer from bacteria to eukaryotes. Nature Reviews Microbiology (2018). doi:10.1038/nrmicro.2017.137

55. Huerta-Cepas, J. et al. EGGNOG 4.5: A hierarchical orthology framework with improved functional annotations for eukaryotic, prokaryotic and viral sequences. Nucleic Acids Res. (2016). doi:10.1093/nar/gkv1248

56. Jones, D. T., Taylor, W. R. \& Thornton, J. M. The rapid generation of mutation data matrices from protein sequences. Bioinformatics (1992). doi:10.1093/bioinformatics/8.3.275

57. Whelan, S. \& Goldman, N. A general empirical model of protein evolution derived from multiple protein families using a maximum-likelihood approach. Mol. Biol. Evol. (2001). doi:10.1093/oxfordjournals.molbev.a003851

58. Kalyaanamoorthy, S., Minh, B. Q., Wong, T. K. F., Von Haeseler, A. \& Jermiin, L. S. ModelFinder: Fast model selection for accurate phylogenetic estimates. Nat. Methods (2017). doi:10.1038/nmeth.4285

59. Lopez, P., Casane, D. \& Philippe, H. Heterotachy, an important process of protein evolution. Mol. Biol. Evol. (2002). doi:10.1093/oxfordjournals.molbev.a003973

60. Ronquist, F. et al. Mrbayes 3.2: Efficient bayesian phylogenetic inference and model choice across a large model space. Syst. Biol. (2012). doi:10.1093/sysbio/sys029

61. Quang, L. S., Gascuel, O. \& Lartillot, N. Empirical profile mixture models for phylogenetic reconstruction. Bioinformatics (2008). doi:10.1093/bioinformatics/btn445

62. Lartillot, N. \& Philippe, H. A Bayesian mixture model for across-site heterogeneities in the amino-acid replacement process. Mol. Biol. Evol. (2004). doi:10.1093/molbev/msh112

63. Felsenstein, J. Cases in which Parsimony or Compatibility Methods will be Positively Misleading. Syst. Biol. (1978). doi:10.1093/sysbio/27.4.401

64. Huelsenbeck, J. P. Is the Felsenstein zone a fly trap? Syst. Biol. (1997). doi:10.1093/sysbio/46.1.69

65. Philippe, H. \& Laurent, J. How good are deep phylogenetic trees? Curr. Opin. Genet. Dev. (1998). doi:10.1016/S0959-437X(98)80028-2

66. Lartillot, N., Brinkmann, H. \& Philippe, H. Suppression of long-branch attraction artefacts in the animal phylogeny using a site-heterogeneous model. in BMC Evolutionary Biology (2007). doi:10.1186/1471-2148-7-S1-S4 
67. Hoang, D. T., Chernomor, O., Von Haeseler, A., Minh, B. Q. \& Vinh, L. S. UFBoot2: Improving the ultrafast bootstrap approximation. Mol. Biol. Evol. (2018). doi:10.1093/molbev/msx281

68. Felsenstein, J. Confidence Limits on Phylogenies: An Approach Using the Bootstrap. Evolution (N. Y). (1985). doi:10.2307/2408678

69. Strimmer, K. \& Rambaut, A. Inferring confidence sets of possibly misspecified gene trees. Proc. R. Soc. B Biol. Sci. (2002). doi:10.1098/rspb.2001.1862

70. Shimodaira, H. An approximately unbiased test of phylogenetic tree selection. Syst. Biol. (2002). doi:10.1080/10635150290069913

71. Susko, E. Tests for two trees using likelihood methods. Mol. Biol. Evol. (2014). doi:10.1093/molbev/msu039

72. Finn, R. D. et al. HMMER web server: 2015 Update. Nucleic Acids Res. (2015). doi:10.1093/nar/gkv397

73. Söding, J. Protein homology detection by HMM-HMM comparison. Bioinformatics (2005). doi:10.1093/bioinformatics/bti125

74. Zimmermann, L. et al. A Completely Reimplemented MPI Bioinformatics Toolkit with a New HHpred Server at its Core. J. Mol. Biol. (2018). doi:10.1016/j.jmb.2017.12.007

75. Doolittle, R. F. Convergent evolution: the need to be explicit. Trends Biochem. Sci. (1994). doi:10.1016/0968-0004(94)90167-8

76. Zhang, J. \& Kumar, S. Detection of convergent and parallel evolution at the amino acid sequence level. Mol. Biol. Evol. (1997). doi:10.1093/oxfordjournals.molbev.a025789

77. Bazykin, G. A. et al. Extensive parallelism in protein evolution. Biology Direct (2007). doi:10.1186/1745-6150-2-20

78. Baalsrud, H. T. et al. De Novo Gene Evolution of Antifreeze Glycoproteins in Codfishes Revealed by Whole Genome Sequence Data. Mol. Biol. Evol. (2018). doi:10.1093/molbev/msx311

79. Bouckaert, R. et al. BEAST 2: A Software Platform for Bayesian Evolutionary Analysis. PLoS Comput. Biol. (2014). doi:10.1371/journal.pcbi.1003537

80. Drummond, A. J., Ho, S. Y. W., Phillips, M. J. \& Rambaut, A. Relaxed phylogenetics and dating with confidence. PLoS Biol. (2006). doi:10.1371/journal.pbio.0040088

81. Drummond, A. J. \& Suchard, M. A. Bayesian random local clocks, or one rate to rule them all. BMC Biol. (2010). doi:10.1186/1741-7007-8-114

82. Betts, H. C. et al. Integrated genomic and fossil evidence illuminates life's early evolution and eukaryote origin. Nat. Ecol. Evol. (2018). doi:10.1038/s41559-018-0644-x

83. Isenbarger, T. A. et al. The most conserved genome segments for life detection on earth and other planets. Orig. Life Evol. Biosph. (2008). doi:10.1007/s11084-008-9148-z

84. Schriek, S., Rückert, C., Staiger, D., Pistorius, E. K. \& Michel, K. P. Bioinformatic evaluation of L-arginine catabolic pathways in 24 cyanobacteria and transcriptional analysis of genes encoding enzymes of L-arginine 
catabolism in the cyanobacterium Synechocystis sp. PCC 6803. BMC Genomics (2007). doi:10.1186/1471-2164-8-437

85. Sánchez-Baracaldo, P., Ridgwell, A. \& Raven, J. A. A neoproterozoic transition in the marine nitrogen cycle. Curr. Biol. (2014). doi:10.1016/j.cub.2014.01.041

86. Erwin, D. H. et al. The Cambrian conundrum: Early divergence and later ecological success in the early history of animals. Science (80-. ). (2011). doi:10.1126/science.1206375

87. Yuan, X., Chen, Z., Xiao, S., Zhou, C. \& Hua, H. An early Ediacaran assemblage of macroscopic and morphologically differentiated eukaryotes. Nature (2011). doi:10.1038/nature09810

88. Uyeda, J. C., Harmon, L. J. \& Blank, C. E. A comprehensive study of cyanobacterial morphological and ecological evolutionary dynamics through deep geologic time. PLoS One (2016). doi:10.1371/journal.pone.0162539

89. Philippe, H. et al. Mitigating Anticipated Effects of Systematic Errors Supports Sister-Group Relationship between Xenacoelomorpha and Ambulacraria. Curr. Biol. (2019). doi:10.1016/j.cub.2019.04.009

90. El-Sayed, A. S. A. et al. Biochemical characterization of peptidylarginine deiminase-like orthologs from thermotolerant Emericella dentata and Aspergillus nidulans. Enzyme Microb. Technol. (2019). doi:10.1016/j.enzmictec.2019.02.004

91. Chavanas, S. et al. Comparative analysis of the mouse and human peptidylarginine deiminase gene clusters reveals highly conserved non-coding segments and a new human gene, PADI6. Gene (2004). doi:10.1016/j.gene.2003.12.038

92. Brahmajosyula, M. \& Miyake, M. Role of peptidylarginine deiminase 4 (PAD4) in pig parthenogenetic preimplantation embryonic development. Zygote (2013). doi:10.1017/S0967199412000160

93. Xiao, S. et al. SMARCAD1 Contributes to the Regulation of Naive Pluripotency by Interacting with Histone Citrullination. Cell Rep. 18, 3117-3128 (2017).

94. Golenberg, N. et al. Citrullination regulates wound responses and tissue regeneration in zebrafish. bioRxiv 2019.12.27.889378 (2019). doi:10.1101/2019.12.27.889378

95. Huang, J. Horizontal gene transfer in eukaryotes: The weak-link model. BioEssays (2013). doi:10.1002/bies.201300007

96. Lim, W. A. \& Pawson, T. Phosphotyrosine Signaling: Evolving a New Cellular Communication System. Cell (2010). doi:10.1016/j.cell.2010.08.023

97. Mikuls, T. R. et al. Porphyromonas gingivalis and disease-related autoantibodies in individuals at increased risk of rheumatoid arthritis. Arthritis Rheum. (2012). doi:10.1002/art.34595

98. Holm, L. \& Rosenström, P. Dali server: Conservation mapping in 3D. Nucleic Acids Res. (2010). doi:10.1093/nar/gkq366

99. Pettersen, E. F. et al. UCSF Chimera - A visualization system for exploratory research and analysis. J. Comput. Chem. (2004). doi:10.1002/jcc.20084

100. Meng, E. C., Pettersen, E. F., Couch, G. S., Huang, C. C. \& Ferrin, T. E. Tools 
for integrated sequence-structure analysis with UCSF Chimera. BMC Bioinformatics (2006). doi:10.1186/1471-2105-7-339

101. Huerta-Cepas, J., Serra, F. \& Bork, P. ETE 3: Reconstruction, Analysis, and Visualization of Phylogenomic Data. Mol. Biol. Evol. (2016). doi:10.1093/molbev/msw046

102. Potter, S. C. et al. HMMER web server: 2018 update. Nucleic Acids Res. (2018). doi:10.1093/nar/gky448

103. Altschul, S. F. et al. Gapped BLAST and PSI-BLAST: A new generation of protein database search programs. Nucleic Acids Research (1997). doi:10.1093/nar/25.17.3389

104. Alva, V., Nam, S. Z., Söding, J. \& Lupas, A. N. The MPI bioinformatics Toolkit as an integrative platform for advanced protein sequence and structure analysis. Nucleic Acids Res. (2016). doi:10.1093/nar/gkw348

105. Remmert, M., Biegert, A., Hauser, A. \& Söding, J. HHblits: Lightning-fast iterative protein sequence searching by HMM-HMM alignment. Nat. Methods (2012). doi:10.1038/nmeth.1818

106. Hildebrand, A., Remmert, M., Biegert, A. \& Söding, J. Fast and accurate automatic structure prediction with HHpred. Proteins Struct. Funct. Bioinforma. (2009). doi:10.1002/prot.22499

107. Eddy, S. R. Accelerated profile HMM searches. PLoS Comput. Biol. (2011). doi:10.1371/journal.pcbi.1002195

108. Steinegger, M. \& Söding, J. MMseqs2 enables sensitive protein sequence searching for the analysis of massive data sets. Nature Biotechnology (2017). doi:10.1038/nbt.3988

109. Trifinopoulos, J., Nguyen, L. T., von Haeseler, A. \& Minh, B. Q. W-IQ-TREE: a fast online phylogenetic tool for maximum likelihood analysis. Nucleic Acids Res. (2016). doi:10.1093/nar/gkw256

110. Rambaut, A. FigTree. version 1.4.3. Institute of Evolutionary Biology, University of Edinburgh (2016).

111. Sangwan, N., Xia, F. \& Gilbert, J. A. Recovering complete and draft population genomes from metagenome datasets. Microbiome (2016). doi:10.1186/s40168-016-0154-5

112. Bernard, G., Greenfield, P., Ragan, M. A. \& Chan, C. X. k -mer Similarity, Networks of Microbial Genomes, and Taxonomic Rank . mSystems (2018). doi:10.1128/msystems.00257-18

113. Letunic, I. \& Bork, P. Interactive tree of life (iTOL) v3: an online tool for the display and annotation of phylogenetic and other trees. Nucleic Acids Res. (2016). doi:10.1093/nar/gkw290

114. Altschul, S. F., Gish, W., Miller, W., Myers, E. W. \& Lipman, D. J. Basic local alignment search tool. J. Mol. Biol. (1990). doi:10.1016/S0022-2836(05)803602

115. Wattam, A. R. et al. Improvements to PATRIC, the all-bacterial bioinformatics database and analysis resource center. Nucleic Acids Res. (2017). doi:10.1093/nar/gkw1017

116. György, B., Tóth, E., Tarcsa, E., Falus, A. \& Buzás, E. I. Citrullination: A 
posttranslational modification in health and disease. International Journal of Biochemistry and Cell Biology (2006). doi:10.1016/j.biocel.2006.03.008

117. McGraw, W. T., Potempa, J., Farley, D. \& Travis, J. Purification, characterization, and sequence analysis of a potential virulence factor from Porphyromonas gingivalis, peptidylarginine deiminase. Infect. Immun. (1999). doi:10.1128/iai.67.7.3248-3256.1999

118. Katoh, K., Rozewicki, J. \& Yamada, K. D. MAFFT online service: Multiple sequence alignment, interactive sequence choice and visualization. Brief. Bioinform. (2018). doi:10.1093/bib/bbx108

119. Nguyen, L. T., Schmidt, H. A., Von Haeseler, A. \& Minh, B. Q. IQ-TREE: A fast and effective stochastic algorithm for estimating maximum-likelihood phylogenies. Mol. Biol. Evol. (2015). doi:10.1093/molbev/msu300

120. Le, S. Q. \& Gascuel, O. An improved general amino acid replacement matrix. Mol. Biol. Evol. (2008). doi:10.1093/molbev/msn067

121. Shimodaira, H. \& Hasegawa, M. Multiple comparisons of log-likelihoods with applications to phylogenetic inference. Molecular Biology and Evolution (1999). doi:10.1093/oxfordjournals.molbev.a026201

122. Shimodaira, H. \& Hasegawa, M. CONSEL: for assessing the confidence of phylogenetic tree selection. Bioinformatics (2001). doi:10.1093/bioinformatics/17.12.1246

123. Guindon, S. et al. New algorithms and methods to estimate maximumlikelihood phylogenies: Assessing the performance of PhyML 3.0. Syst. Biol. (2010). doi:10.1093/sysbio/syq010

124. Anisimova, M., Gil, M., Dufayard, J. F., Dessimoz, C. \& Gascuel, O. Survey of branch support methods demonstrates accuracy, power, and robustness of fast likelihood-based approximation schemes. Syst. Biol. (2011). doi:10.1093/sysbio/syr041

125. Lartillot, N., Rodrigue, N., Stubbs, D. \& Richer, J. Phylobayes mpi: Phylogenetic reconstruction with infinite mixtures of profiles in a parallel environment. Syst. Biol. (2013). doi:10.1093/sysbio/syt022

126. Di Tommaso, P. et al. T-Coffee: A web server for the multiple sequence alignment of protein and RNA sequences using structural information and homology extension. Nucleic Acids Res. (2011). doi:10.1093/nar/gkr245

127. Söding, J., Biegert, A. \& Lupas, A. N. The HHpred interactive server for protein homology detection and structure prediction. Nucleic Acids Res. (2005). doi:10.1093/nar/gki408

128. Edgar, R. C. MUSCLE: Multiple sequence alignment with high accuracy and high throughput. Nucleic Acids Res. (2004). doi:10.1093/nar/gkh340

129. Waterhouse, A. M., Procter, J. B., Martin, D. M. A., Clamp, M. \& Barton, G. J. Jalview Version 2-A multiple sequence alignment editor and analysis workbench. Bioinformatics (2009). doi:10.1093/bioinformatics/btp033

130. Henikoff, S. \& Henikoff, J. G. Amino acid substitution matrices from protein blocks. Proc. Natl. Acad. Sci. U. S. A. (1992). doi:10.1073/pnas.89.22.10915

131. Jones, D. T. Protein secondary structure prediction based on position-specific scoring matrices. J. Mol. Biol. (1999). doi:10.1006/jmbi.1999.3091 
132. Drummond, A. J. \& Rambaut, A. BEAST: Bayesian evolutionary analysis by sampling trees. BMC Evol. Biol. (2007). doi:10.1186/1471-2148-7-214

133. Kumar, S. \& Hedges, S. B. Timetree2: Species divergence times on the iPhone. Bioinformatics (2011). doi:10.1093/bioinformatics/btr315

134. Timmis, J. N., Ayliff, M. A., Huang, C. Y. \& Martin, W. Endosymbiotic gene transfer: Organelle genomes forge eukaryotic chromosomes. Nature Reviews Genetics (2004). doi:10.1038/nrg1271

135. Shapiro, S. S. \& Wilk, M. B. An Analysis of Variance Test for Normality (Complete Samples). Biometrika (1965). doi:10.2307/2333709

136. Yilmaz, P. et al. The SILVA and 'all-species Living Tree Project (LTP)' taxonomic frameworks. Nucleic Acids Res. (2014). doi:10.1093/nar/gkt1209

137. Needleman, S. B. \& Wunsch, C. D. A general method applicable to the search for similarities in the amino acid sequence of two proteins. J. Mol. Biol. (1970). doi:10.1016/0022-2836(70)90057-4 


\section{Materials and Methods:}

\section{Structural analyses}

Structural homology searches were performed using the Dali server v3.1 with the extracted PAD_C domain used as query ${ }^{98}$. Superposition of known structures was performed in $\bar{C}$ imera ${ }^{99}$ using the MatchMaker tool ${ }^{100}$. Briefly, the two structures (PDB: $4 \mathrm{n} 2 \mathrm{c}$ and $1 \mathrm{xkn}$ ) were aligned for the best-aligning pair of chains using the Needleman-Wunsch algorithm and BLOSUM62 matrix. A secondary structure score of $30 \%$ was included. The superposition was iterated by pruning long atom pairs such that no pair exceeds 2.0 angstroms.

\section{Identification of PADI orthologues}

A graph-based unsupervised clustering algorithm used by the EggNOG database was used to infer PADI orthologous groups from 2031 genomes across the tree of life (ENOG410ZKF3: 217 proteins from 74 species) ${ }^{55}$. Phylogenetic reconstruction for the identified PADI orthologues was performed within EggNOG as implemented within the ETE3 suite (eggnog41) and described at http://eggnogdb.embl.de/\#/app/methods ${ }^{55,101}$. In addition, a list of proteins with significant similarity (E-value $<1 \times 10^{-3}$ ) to the metazoan PAD_C domain from human PADI2 were collected using HMMER searches against Reference Proteomes and UniProtKB databases ${ }^{102}$. Additional more sensitive sequence searches and iterative searches were performed using tblastn and psiblast against $\mathrm{nr} / \mathrm{nt}$; jackhammer against reference proteomes and UniProtKB; and hhpred against Pfam-A, COG_KOG and PDB_mmCIF70 $73,103,104$. To verify the exhaustive nature of our search for PADI homologues we employed two state of the art remote homology detection tools. The first, HHBlits, is used to search databases of hidden markov models $(\mathrm{hmm})$ generated with clustered proteomic datasets with a query $\mathrm{hmm}$ and is available in the HHSuite ${ }^{105,106}$. The second tool, Hmmsearch, is included in the Hmmer suite ${ }^{107}$ and used to search proteomic datasets with an hmm. A PADI alignment was generated from our initial dataset of known homologues for use with both tools using clustal omega on default parameters for 3 iterations to generate our query $\mathrm{hmm}$. HHBlits was used to search the Uniclust30 database. Its construction and contents are detailed on the mmseqs website ${ }^{108}$ (Uniclust. [cited 5 May 2020]. Available: https://uniclust.mmseqs.com/). Hmmsearch was used to search the NCBI NR protein database. Its contents and construction are detailed on the NCBI web page (Download-NCBI. [cited 5 May 2020]. Available: https://www.ncbi.nlm.nih.gov/home/download/). The HHBlits search results were filtered with a cutoff of $90 \%$ probability and 50 amino acids. No additional hits were found to sequences in clades that were not in the starting dataset.

Analysis of spurious viral and archaeal hits: The HMMsearch results were filtered with an E-value cutoff of $10^{-10}$ and 50 amino acids. Two sequences attributed to unexpected clades were found: refseq identifiers AXN91134.1 and RCV64870.1 which are found in Namao virus and Methanophagales archaeon, respectively. The two anomalous sequences are the only representatives of the PADI family within their taxonomic kingdoms and this extremely sparse distribution of these sequences would either imply many independent gene loss events or an extremely recent horizontal transfer event of $P A D I$ to these clades if the genes are in fact correctly 
attributed to their genome. To verify the validity of the attribution of these sequences to their respective genomes, we calculated a phylogeny including by aligning a subset of high confidence PADI sequences with the two putative homologues. The alignment was then used with IQTree ${ }^{109}$ on default parameters and automatic selection of the appropriate model to generate a phylogeny. The resulting tree was visualized with figtree ${ }^{110}$ (Figure S2). The placement of the two sequences in the phylogeny does not agree well with a plausible evolutionary scenario considering their taxonomic origin; rather their placement suggests that the allegedly Methanophagales archaeon sequence is in fact a cyanobacterial sequence, and that the allegedly Namao virus sequence, is in fact a fish sequence. These hypotheses are corroborate by the origin of the samples used to obtain them: metagenomic isolates in the case of Methanophagales archaeon, and infected tissue samples taken from fish in the case of Namao virus. In both cases, the sample were susceptible to gene misattribution due to incorrect binning ${ }^{111}$ or contamination. To further probe the association of these genes to their respective genomes and discount the possibility of the PADI genes belonging to a transferred genomic segment, we used the K-mer spectra of the genomes to study the possibility of horizontal transfer events. These analytics are regularly used to find transferred regions in prokaryotic genomes ${ }^{112}$. Normalized K-mer spectra for DNA sequences were generated by counting occurrences of all K-mers and normalizing by the total amount of words counted to give a unit vector. The results presented in Figure S3 were derived using 4-mers. To detect possible horizontally transferred genomic regions, an average spectrum for the entire genome was calculated. A spectrum was then calculated for a sliding window of $1 \mathrm{~kb}$ using $500 \mathrm{bp}$ steps and subtracted from the genomic average at each window position. The absolute value of the difference between the genomic average and window spectra is represented over the entire genome. The code for running these kmer-based analyses is available at https://github.com/DessimozLab/Pad1.

\section{Phylogenetic methods}

For all phylogenetic trees, branch support information was visualised and figures produced using FigTree $\mathrm{v} 1.4 .3$ and $\mathrm{iTOL}^{113}$. Amino acid sequences for PADI homologues were obtained from UniProtKB, NCBI and Pathosystems Resource Integration Center (PATRIC) databases using HMMER and BLAST searches ${ }^{72,114,115}$. PADI2 was used for species with multiple PADI paralogues, as it closest resembles the PADI gene in metazoa with one PADI (such as fish ${ }^{116}$ ), and with the PADI2 from metazoan species with three PADIs such as birds or reptiles (Figure S7).

Phylogenetic analysis of other citrullinating enzymes: Sequences of the arginine deiminase from Giardia lamblia $\left.(\mathrm{gADI})^{33}\right)$ and the porphyromonas-type peptidylarginine deiminase from Porphyromonas gingivalis $\left(\mathrm{pPAD}^{117}\right)$ were used as a seed for Hidden Markov Model (HMM) searches of reference proteomes to identify sequences from other species of similar length and most significant similarity ${ }^{72,102}$. These were aligned with 25 representative PADI sequences using MAFFT L-ins-I ${ }^{118}$ and singly aligning columns were removed. IQTree was used to produce a maximum likelihood phylogenetic tree ${ }^{109,119}$. The LG empirical rate matrix with 8 categories of rate variation under the FreeRate model $(L G+R 8)$ was used, as determined by ModelFinder $r^{58,120}$ according to the corrected Akaike Information Criterion. The 
Ultrafast Bootstrap 2 with 1000 replicates ${ }^{67}$, Shimodaira-Hasegawa (SH)-like approximate likelihood-ratio test (aLRT) with 1000 replicates $^{121-123}$, and aBayes parametric tests ${ }^{124}$ were used to assess node support.

Phylogenetic analysis of PADI orthologues: All putative bacterial PADI sequences in the PATRIC database were obtained from BLAST searches ${ }^{72,114,115}$. In addition, sequences from metazoa were subsampled to maximise the inclusion of different lineages. The human PADI2 sequence was searched against UniProtKB to subsample sequences from 35 fungal species that represent the broadest distribution of affinity with PADI2 as determined by HMMER bitscore ${ }^{102}$. This was done to provide representation from the maximum sequence diversity of fungal PADIs. Fragment sequences were excluded. The collected sequences were aligned using MAFFT L-ins-I and singly aligning columns were removed ${ }^{118}$. IQTree was used to produce a maximum likelihood phylogenetic tree ${ }^{109,119}$. The WAG empirical rate matrix with 10 categories of rate variation under the FreeRate model with base frequencies counted from the alignment $(W A G+R 10+F)$ was used as determined by ModelFinder according to the corrected Akaike Information Criterion ${ }^{57,58}$. Ultrafast Bootstrap 2 with 1000 replicates, SH-like aLRT with 1000 replicates, and aBayes parametric tests were used to assess node support ${ }^{67,121-124}$. The tree is shown rooted at the midpoint with solid circles indicating consensus node support of $>95 \%$. A number of critical nodes for testing different evolutionary hypotheses were labelled in full as they are mentioned specifically in the text or referred to in other analyses.

Phylogenetic analysis of subsampled PADI orthologues for topology testing: Bacterial PADI sequences were subsampled to maximise sequence diversity. Both the closest and the most distant bacterial homologues with respect to the metazoan sequence were retained to allow for the broadest distribution of protein sequences (to a total of 50 sequences). Firstly, a maximum likelihood phylogenetic tree was produced using IQTree as above using the best empirical rate matrix according to ModelFinder (WAG + R5 +F0). This reproduced the topology obtained with the full tree. Three further analyses were performed:

1) Maximum likelihood phylogenetic analysis was performed using IQTree under the C20 empirical profile mixture model of evolutionary rate matrices using CIPRES Gateway on XSEDE ${ }^{61}$.

2) Bayesian phylogenetic inference was performed using MrBayes v3.2.6 x64 using CIPRES Gateway on XSEDE with mixed model MCMC jumping across different fixed empirical rate matrices and 5 different gamma distributed rate categories ${ }^{60}$. Analysis was performed with 4 runs each of 1000000 chains. The average standard deviation of split frequencies was observed to be $<0.005$, parameters all had an effective sample size (ESS) > 500 and potential scale reduction factor (PSRF) of 1.000 (to 3 significant figures). The summary tree was generated with a burn-in of $25 \%$ over the runs. Posterior probability was used for node support- i.e. where posterior probability was 100 , the topology was congruent in every tree sampled by the Markov chain Monte Carlo (MCMC) after burn-in. The aminoacid model prior was set as 'mixed' such that the MCMC jumps across different models i.e. mixture of models with fixed rate matrices. Poisson, Jones, Dayhoff, Mtrev, Mtmam, Wag, Rtrev, Cprev, Vt and Blosum models were used and all have equal prior probability. The WAG model had posterior probability of 1.000 , and standard deviation $<0.0001$ - and was exclusively sampled from the posterior ${ }^{57}$. This is consistent with the WAG model being identified 
as the best empirical matrix identified according to ModelFinder and the corrected Akaike information criterion from the maximum likelihood analysis in IQTree.

3) Bayesian phylogenetic inference was performed using PhyloBayes under the CAT-GTR model ${ }^{62,125}$. This is an infinite mixture model of rate matrices making use of a Dirichlet process prior. The alignment contains 1100 aligned positions and 50 taxa. 8 chains were performed in parallel for 24 hours such that more than 20000 cycles were achieved as recommended in the PhyloBayes manual using the MRC IGMM and University of Edinburgh computing cluster Eddie3. Readpb, bpcomp, tracecomp tools in PhyloBayes and Tracer software were then used to analyse runs. Posterior consensus trees were generated for each run and were reproducible across the eight different runs. The trace plots for independent runs were also analysed to assess for apparent stationarity aiming for an ESS of at least 100. Maxdiff was observed to be < $0.1($ maxdiff $=0.06209$, meandiff $=0.00330)$.

Tree topologies were congruent across the four different methods (Figure 1b). Additional topology testing was performed in PAUP*4.0a163 and in IQTree. 100 random trees were generated along with the maximum likelihood constrained tree where fungal and metazoan sequences were constrained to be monophyletic. The $\mathrm{SH}$-test, approximately unbiased (AU) test and expected likelihood weight (ELW) tests were performed and all other alternative trees, including the constraint tree were rejected $(p<0.0001)^{69-71,122}$.

Phylogenetic analysis excluding synapomorphic regions: Phylogenetic analyses from Figure 1 were repeated using an alignment with the PAD_N domain removed and with an alignment in which both the PAD_N domain and regions of synapomorphy (Figure S4) were removed. Maximum likelihood analysis using IQTree with ModelFinder used to select the best performing fixed empirical rate matrix (WAG + R5 +F0) as above ${ }^{58,67,109,119}$. Topologies were congruent with analysis of the whole alignment and node support values (Ultrafast Bootstrap 2) for the clades labelled in Figure 1 are provided in Figure S4b.

\section{PADI Domain annotation}

To identify putative locations for the three PAD domains within PADI homologue sequences from bacteria and fungi, each target PADI sequence was aligned to five metazoan sequences using TCoffee ${ }^{126}$. Putative domain sequence regions were then used as a target query for HMMER or HHPred searches ${ }^{72,127}$. HMMER searches were made against the UniProtKB database and HHPred searches were made, firstly against a database of HMM profiles of protein domains in the Protein Data Bank (PDB_mmCIF_4_Aug) and secondly, against a database of profiles from Pfam (Pfam-A_v31.0 $)^{104}$. Once individual sequences were identified as possessing a specific domain architecture, multiple sequence alignments of groups of sequences with common putative domain architecture were made and this was used as a query for each type of search.

For the reported E-values in Figure S4a the following method was used. All sequences from the highlighted clade in the phylogenetic tree were aligned using TCoffee, PAD_C, PAD_M and PAD_N domains extracted from the cyanobacterial sequences, and secondly PAD_C and PAD_M domains from the clade containing a mixture of bacterial and fungal sequences. This alignment was used as a seed for searches with HHPred against a database of profiles made of the entire human 
proteome, and against a database of profiles of Pfam domains (Pfam-A_v31.0). HHPred searches were performed using the MPI Bioinformatics Toolkit of the Max Planck Institute for Developmental Biology, Tübingen, Germany ${ }^{74,104}$.

\section{Multiple sequence alignment of $P A D \_N$ domain}

Amino acid sequences were aligned using MUSCLE or TCoffee algorithms ${ }^{126,128}$ and visualised using Jalview ${ }^{129}$. Putative PAD_N domains from SPMINX clade cyanobacterial PADI sequences were identified using HHPred showing significant statistical evidence for affinity (E-value: $\left.2.5 \times 10^{-5}\right)^{74,104}$. These were aligned with the PAD_N domain from human PADI paralogues and Rhincodon typus (whale shark). The alignment was presented with the program Belvu using a colouring scheme indicating the average BLOSUM62 scores (which are correlated with amino acid conservation) of each alignment column ${ }^{130}$, as represented in Figure 2a. PsiPred ${ }^{131}$ was used to predict secondary structure for the cyanobacterial PAD_N domains (beta sheets). The secondary structure of the PAD_N domain of human PADI2 was identified from the crystal structure (PDB: $4 \mathrm{n} 2 \mathrm{a})^{28}$.

\section{Synapomorphy analysis of PADI calcium binding sites}

Representative fungal, actinobacterial, cyanobacterial, and metazoan PADI sequences were analysed for the conservation of all of the calcium-binding sites (a minimum of three residues coordinate each calcium binding site) and for other critical residues contained at the active site (Figure 2). PADIs from the following species were used: 1) metazoan PADIs from Homo sapiens, Xenopus laevis, Oncorhynchus mykiss, Callorhinchus milii, Branchiostoma floridae, Priapulus caudatus; 2) cyanobacterial PADIs from Cyanothece sp. 8801, Stanieria cyanosphaera, Chlorogloeopsis fritschii PCC 6912, Crocosphaera subtropica, Aphanothece sacrum, Cyanothece sp. 7424; 3) fungal PADIs from Fusarium sp. FOSC 3-a, Periconia macrospinosa, Paracoccidioides lutzii, Blastomyces parvus, Ajellomyces capsulatus, Emmonsia crescens and; 4) actinobacterial PADIs from Streptomyces silvensis, Alteromonas lipolytica, Streptomyces sp. 3214.6, Erythrobacter xanthus, Kibdelosporangium aridum, Nocardia brasiliensis ATCC 700358. Sequences were aligned using MAFFT L-ins-I and compared to functionally annotated regions from Slade et al. 2015 and from crystal structures ${ }^{28,34,118}$.

\section{Sequence divergence analyses}

BEAST v2.4.8 was used to produce a time tree of the clade of subsampled metazoan PADIs and the full clade of closest SPMINX cyanobacteria contained within the PATRIC database using the GTR model with 4 gamma distributed rate categories $^{79,88,132}$. The following metazoan species were used: Homo sapiens (HS), Mus musculus (MM), Alligator mississippiensis (AM), Chelonia mydas (CM), Gallus gallus (GG), Xenopus laevis (XL), Oncorhynchus mykiss (OM), Callorhinchus milii (CM), Branchiostoma floridae (BF), Priapulus caudatus (PC). To calibrate nodes on the tree, node times were set as the following normally distributed priors: mean 797.0, sigma 72.5 (clade of HS, MM, AM, CM, GG, XL, OM, CM, BF, PC); mean 692.5, sigma 57.5 (clade of HS, MM, AM, CM, GG, XL, OM, CM, BF); mean 473.5, 
sigma 14.0 (clade of HS, MM, AM, CM, GG, XL, OM, CM); mean 435.0, sigma 6.5 (clade of HS, MM, AM, CM, GG, XL, OM); mean 311.0, sigma 7.5 (clade of HS, MM, AM, CM, GG); mean 89.5, sigma 3.0 (clade of HS, MM). Metazoan divergence times from the fossil record were obtained from timetree.org with bounds on the distributions chosen to span the range of times reported in the literature centered on the median value ${ }^{133}$. The calibrated Yule model was used as the tree prior. XML files were generated in BEAUti and the MCMC analysis was run using BEAST2 on the CIPRES Gateway on XSEDE. An initial MCMC run of 5,000,000 chains was run for each clock model ${ }^{80,81}$. Then full analysis was performed with two independent runs of $10,000,000$ chains for the strict clock, uncorrelated lognormal (UCLN) and uncorrelated exponentially distributed (UCED) clock models and three independent runs of $10,000,000$ chains for the random local clocks model. All models were additionally run under the tree prior (i.e. in the absence of sequence data) and assessed. Analysis of parameters was performed in Tracer to assess apparent stationarity for the different tree parameters and for acceptable ESS values and congruence was assessed across the independent runs. The predicted divergence time of the metazoan and cyanobacterial clades was given by the marginal posterior distribution of the age of the root of the whole tree. This is given by the TreeHeight parameter. The $95 \%$ confidence interval of the TreeHeight parameter for all runs under each of the clock models did not exceed 1300. This data was plotted as a violin plot showing 95\% confidence intervals (Figure $3 \mathrm{~b}$ ).

\section{Accumulated genetic divergence analysis relative to other proteins}

Bitscore density is calculated by taking the bitscore of a query sequence to the target sequence produced by HMMER and dividing by the bitscore of the query sequence to itself (longer sequences have higher bitscores), which gives a value between 0 and $1^{72}$. The bitscore densities of the similarity of 1 ) the cyanobacterial homologue to the human sequence: $\Delta$ bitscore $_{\mathrm{Cy}-\mathrm{Hu}}(\mathrm{AC}+\mathrm{AH})$ and 2$)$ of the branchiostomal

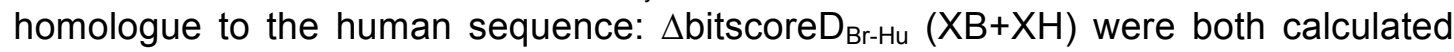
(Figure S5). A measure of the total accumulated genetic divergence between latediverging cyanobacteria (Cyanothece spp.) and the last common ancestor of Branchiostoma spp. and Homo sapiens was then calculated by subtracting

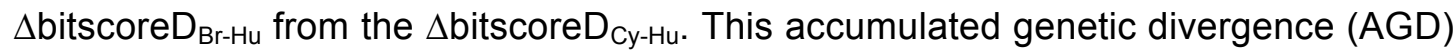
value was calculated for: (1) 26 ribosomal proteins (uS2, uS3, uS4, uS5, uS7, uS8, uS9, uS10, uS11, uS12, uS13, uS17, uS19, uL1, uL2, uL3, uL4, uL5, uL6, uL11, uL13, uL14, uL15, uL22, uL23, uL24), (2) 19 sequences whose proteins are mitochondrially located so are reasonable EGT candidates from the mitochondrion (OTC, ASS1, ARLY, CPS1, PGK, ENO, GAPDH, PK, NAXE, G6PD, RPIA, FUMH, SDHB, SDHA, CS, MDHM, DLAT, DLDH, ACLY) ${ }^{134}$, and (3) all 10 proteins still encoded in the mitochondrial genome (MT-ATP6, MT-CO1, MT-CO2, MT-CO3, MTCYB, MT-1, MT-2, MT-3, MT-4, MT-5). It is notable that by definition, only very highly conserved proteins have an AGD that can be calculated in this extreme example between the last common ancestor of late diverging cyanobacteria and humans: if a protein has diverged substantially then the similarity of the human homologue to the cyanobacterial will not be discernible and no bitscore can be calculated. AGD values of proteins in each category were then tested for deviation from a normal distribution using the Shapiro Wilk test $\left(W=b^{2} / S S\right)^{135}$. As the calculated $p$-value $>0.05$, the null hypothesis was retained and the data treated as being normally distributed. Kurtosis and skew were also within the range of the normal distribution. The AGD for PADI 
proteins $\left(A G D_{\text {PADI proteins }}=0.066\right)$ was then compared to the mean $A G D$ of each category of control proteins (e.g. $A G D_{\text {ribosomal proteins }}=0.70$ ) and z-scores were calculated.

To compare the extend of divergence relative to ribosomal RNA (rRNA), nucleotide sequences for rRNA were obtained from the SILVA database ${ }^{136}$. Nucleotide sequences for PADIs were obtained from NCBI and exons extracted. Comparisons were made with EMBOSS Needle using the Needleman-Wunsch global alignment algorithm $^{137}$ (gap open: 10, gap extend: 0.5 ).

\section{Preparation of recombinant proteins}

PADI gene sequences were obtained from NCBI and synthesised by Thermo GeneArt with flanking EcoRI (at the 5' end) and Xhol (at the 3' end) restriction sites. Cyanothece sp. $8801 \mathrm{PADI}$ and human PADI4 sequences were subcloned into a modified pGEX vector, which included an additional 10X His tag immediately $\mathrm{N}$ terminal of the enzyme sequence (generous gift from Dr Martin Reijns, MRC Human Genetics Unit), by InFusion cloning. GST-His-PADI4 and GST-His-cyanoPADI were expressed in BL21 (DE3) in $2 \mathrm{TY}$ cultures. Cells were grown $\left(37^{\circ} \mathrm{C} ; 180 \mathrm{rpm}\right)$ to an OD600 of 0.6 and induced overnight at $18{ }^{\circ} \mathrm{C}$ with $0.5 \mathrm{mM} \quad \beta-\mathrm{D}-1-$ thiogalactopyranoside (IPTG). Bacterial pellets were harvested by centrifugation $(8,000 \mathrm{xg} ; 10 \mathrm{~min})$ and frozen at $-80^{\circ} \mathrm{C}$. Cell pellets were resuspended in $50 \mathrm{mM}$ Tris $\mathrm{pH} 7.5,500 \mathrm{mM} \mathrm{NaCl}, 20 \mathrm{mM}$ imidazole, $5 \%$ Glycerol, $1 \mathrm{mM}$ DTT $(1 \mathrm{~g}$ dry cell mass in $4 \mathrm{~mL}$ ), $1 X$ EDTA-free protease inhibitors (Roche), $5 \mathrm{mM} \mathrm{MgCl}_{2}$ and 10 units benzonase at $4{ }^{\circ} \mathrm{C}$ with stirring. Cells were lysed on ice by sonication $(7 \times 45 \mathrm{sec}$, with $45 \mathrm{sec}$ breaks) and the lysate was cleared by centrifugation $(20,000 \mathrm{xg} ; 20 \mathrm{~min})$. Supernatant was sterile filtered $(0.2 \mu \mathrm{m})$ before loading by Superloop onto a $5 \mathrm{~mL}$ HisTrap column, which was pre-equilibrated with binding buffer. Proteins were purified using an AKTA FPLC system (GE Healthcare). The column was washed with $50 \mathrm{mM}$ Tris $\mathrm{pH} 7.5,500 \mathrm{mM} \mathrm{NaCl}, 25 \mathrm{mM}$ imidazole, 5\% Glycerol, $1 \mathrm{mM}$ DTT and the recombinant proteins were eluted with $50 \mathrm{mM}$ TRIS pH 7.5, $500 \mathrm{mM} \mathrm{NaCl}, 250$ $\mathrm{mM}$ imidazole, $5 \%$ Glycerol, $1 \mathrm{mM}$ DTT. The purified sample was concentrated using Vivaspin MWCO filters into $50 \mathrm{mM}$ HEPES $\mathrm{pH} 7.5,150 \mathrm{mM} \mathrm{NaCl}, 5 \mathrm{mM}$ DTT, $5 \%$ $(\mathrm{v} / \mathrm{v})$ glycerol, and the concentration determined using Nanodrop.

\section{Citrullination activity assays}

Using mouse cell lysates: E14 mouse embryonic stem (ES) cells were cultured in GMEM supplemented with $10 \%$ fetal calf serum (FCS), $0.1 \mathrm{mM}$ non-essential amino acids, $2 \mathrm{mM}$ L- glutamine, $1 \mathrm{mM}$ sodium pyruvate, $0.1 \mathrm{mM}$ beta-mercaptoethanol and $10^{6}$ units/L leukaemia inhibitory factor (LIF) (ESGRO, Millipore) and grown on a six well plate until $70 \%$ confluent. Cells were harvested in $0.1 \%$ NP- $40,20 \mathrm{mM}$ Tris pH 7.6, 1X EDTA-free protease inhibitors, $5 \mathrm{mM}$ DTT, after two washes in PBS (one in PBS containing $2 \mathrm{mM}$ EDTA and one in plain PBS). To shear chromatin and clarify lysates, benzonase and $2 \mathrm{mM} \mathrm{MgCl} 2$ were added and samples were rotated at $4^{\circ} \mathrm{C}$ for 30 mins, sheared by passing through a $25 \mathrm{G}$ needle and centrifuged at $13000 \mathrm{rpm}$ for 5 mins. Citrullination activity assays were performed with $500 \mathrm{nM}$ recombinant enzyme in $50 \mathrm{mM}$ HEPES pH 7.5, $150 \mathrm{mM} \mathrm{NaCl}, 5 \mathrm{mM}$ DTT, $5 \%$ (v/v) glycerol, either in the presence of $5 \mathrm{mM} \mathrm{CaCl}_{2}$ or water. Reactions were incubated for 30 mins 
at $37^{\circ} \mathrm{C}$ and quenched by boiling at $95^{\circ} \mathrm{C}$ for 5 mins. Samples were stored at $-80^{\circ} \mathrm{C}$ before immunoblotting.

Using recombinant histone $\boldsymbol{H} 3$ substrate: Reactions were performed in $50 \mathrm{mM}$ HEPES, $137 \mathrm{mM} \mathrm{NaCl}, 5 \mathrm{mM}$ DTT with $1.5 \mu \mathrm{M}$ recombinant $\mathrm{H} 3$ (New England Biolabs), vehicle or $500 \mathrm{nM}$ recombinant enzyme, and in the presence of either $5 \mathrm{mM}$ $\mathrm{CaCl}_{2}$ or water. Reactions incubated for 30 mins at $15^{\circ} \mathrm{C}$ or $37^{\circ} \mathrm{C}$ and quenched by boiling at $95^{\circ} \mathrm{C}$ for 5 mins before immunoblotting.

\section{Immunoblotting}

Proteins were separated by SDS-PAGE and transferred to nitrocellulose membrane using wet transfer. Membranes were blocked in 5\% BSA in TBS containing $0.1 \%$ Tween-20 for $1 \mathrm{~h}$ at room temperature. Proteins were detected using primary antibodies against anti-H3 (Abcam ab10799, 1:1000), anti-H3CitR2 (Abcam ab176843, 1:2000), anti-NPM1 (Abcam ab37659, 1:200) and anti-GST (Abcam ab19256, 1:1000) overnight at $4{ }^{\circ} \mathrm{C}$ and in secondary antibody at 1:5000 for $1 \mathrm{~h}$ at room temperature. Membranes were incubated in Pierce ECL reagent and imaged using ImageQuant LAS 4000 (GE). Citrulline-containing proteins were modified on the membrane and detected using the anti-modified citrulline detection kit (Millipore, 17-347) according to manufacturer's instructions. 
bioRxiv preprint doi: https://doi.org/10.1101/2020.06.13.150037· this version posted June 15,2020 . The copyright holder for this preprint (which was not certified by peer review) is the author/funder, who has granted bioRxiv a license to display the preprint in perpetuity. It is

Table 1 made available under aCC-BY-NC-ND 4.0 International license.

\begin{tabular}{|c|c|c|c|c|}
\hline Group & $\begin{array}{l}\text { NCBI } \\
\text { Taxonomy ID }\end{array}$ & $\begin{array}{l}\text { Unique species } \\
\text { with a PADI }\end{array}$ & $\begin{array}{l}\text { Species with } \\
\text { proteomes in } \\
\text { UniprotKB }\end{array}$ & $\begin{array}{l}\text { Percentage of } \\
\text { species with a } \\
\text { PADI }\end{array}$ \\
\hline Bacteria & 2 & 295 & 38842 & 0.76 \\
\hline Cyanobacteria & 1117 & 56 & 506 & 11.07 \\
\hline Actinobacteria & 201174 & 136 & 4870 & 2.79 \\
\hline Proteobacteria & 1224 & 69 & 16196 & 0.43 \\
\hline Eukaryotes & 2759 & 406 & 2241 & 18.12 \\
\hline Animals (Metazoa) & 33208 & 229 & 612 & 37.42 \\
\hline Insects & 50557 & 0 & 142 & 0.00 \\
\hline Worms (Annelida) & 6340 & 0 & 2 & 0.00 \\
\hline Fungi & 4751 & 177 & 1098 & 16.12 \\
\hline Yeast (Ascomycota) & 4890 & 176 & 760 & 23.16 \\
\hline Yeast (Saccharomyces) & 4930 & 0 & 13 & 0.00 \\
\hline Plants (Viridiplantae) & 33090 & 0 & 244 & 0.00 \\
\hline Opisthokonta (metazoa and fungi) & $33208 \& 4751$ & 406 & 1710 & 23.74 \\
\hline Pre-opisthokonta (Eukarya, not metazoa or fungi) & $\begin{array}{r}2759 \text { \& NOT } \\
(33208 \mid 4751)\end{array}$ & 0 & 531 & 0.00 \\
\hline Archaea & 2157 & 1 & 2107 & 0.05 \\
\hline Viruses & 10239 & 1 & 99210 & 0.001 \\
\hline
\end{tabular}

Table 1: The number and proportion of species harbouring a putative PADI orthologue.

HMM searches (https://www.ebi.ac.uk/Tools/hmmer) for similarity to the vertebrate PAD_C domain from human PADI2, were carried out using HmmerWeb version 2.41.1 against the UniProtKB (v.2019_09)

database. Unique species with significant sequence similarity (E-value $<1 \times 10-3)$ are presented.

Proportions are given relative to the total number of species in within UniProtKB, for each group. 


\section{a}

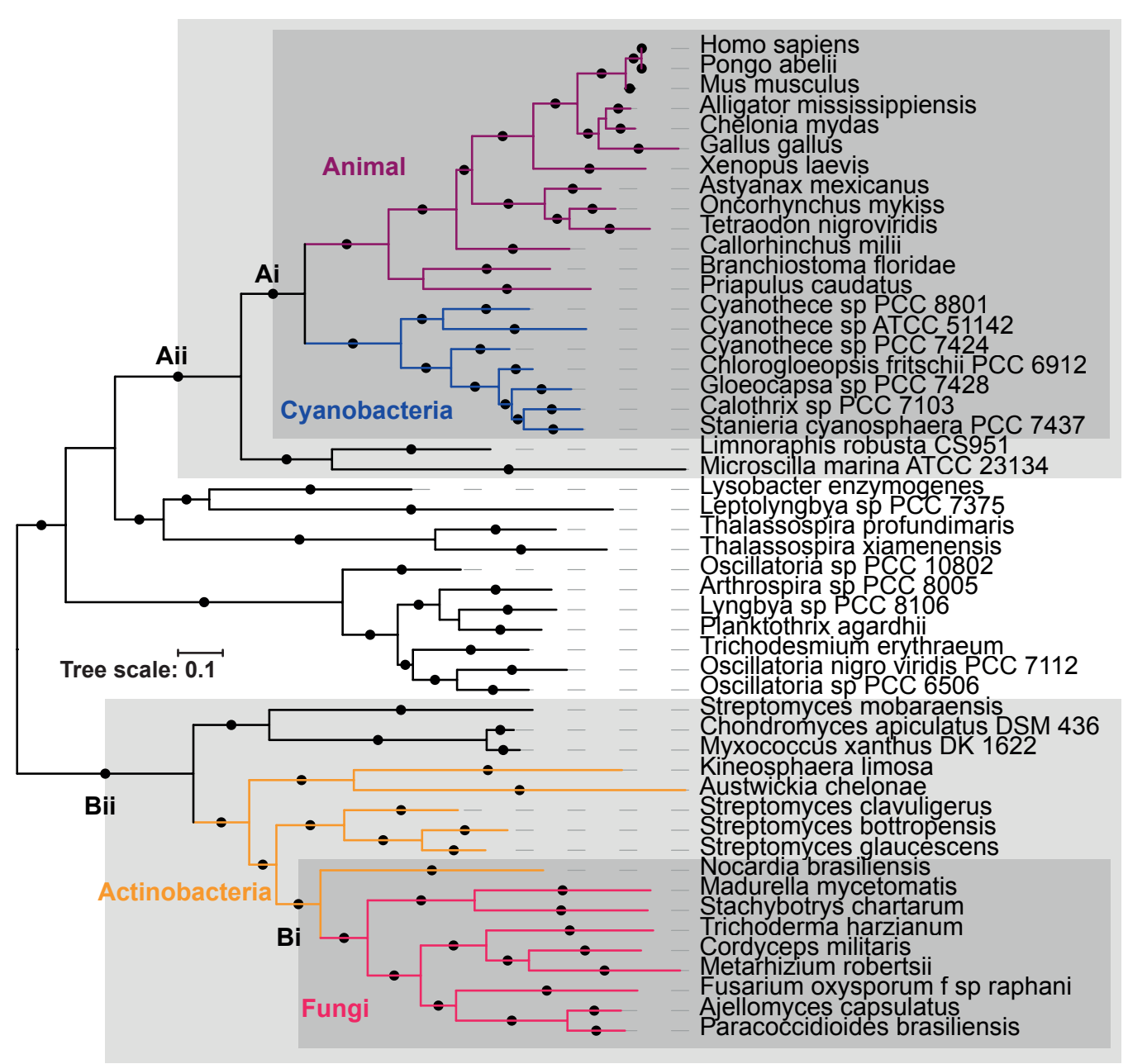

b

Node support for clade:

\begin{tabular}{|l|cccc|}
\cline { 2 - 5 } \multicolumn{1}{l|}{ Phylogenetic method: } & Ai & Aii & Bi & Bii \\
\hline IQTree WAG+R5+F0 & 96 & 100 & 100 & 100 \\
\hline MrBayes mixed model jump & 100 & 100 & 100 & 100 \\
\hline IQTree C20 mixture model & 99 & 100 & 99 & 100 \\
\hline PhyloBayes CAT-GTR & 99 & 100 & 100 & 100 \\
\hline
\end{tabular}

Figure 1: Phylogeny of the PADI sequence. a) Consensus topology for all phylogenetic methods with branch lengths from Bayesian phylogenetic inference with MrBayes. Solid circles indicate consensus node support of $>95 \%$. b) Node support for analysis with: 1) maximum likelihood inference using WAG fixed rate matrix, 2) MrBayes sampling across different fixed rate matrices, 3) IQTree using the C20 mixture model of rate matrices and 4) PhyloBayes using the CAT-GTR model of an infinite mixture model of rate matrices. Ultrafast bootstrap 2 values over 1000 replicates (for the Maximum likelihood methods) or posterior probabilities (for the Bayesian methods) are presented in the table for the nodes labelled in the tree critical to different evolutionary scenarios. 
a

Cyanothece
Nostoc
Anabaena
Calothrix2100
Calothrix2098
Stanieria
Calothrix7103
Gloeocapsa
Desertifilum
Leptolyngbya
Cyanothece7424
Cyanobacterium
PsiPred 2D
PADI_WhaleShark
PADI1_HUMAN
PADI3_HUMAN
PADI6_HUMAN
PADI4_HUMAN
PADI2_HUMAN
PADI2 2D

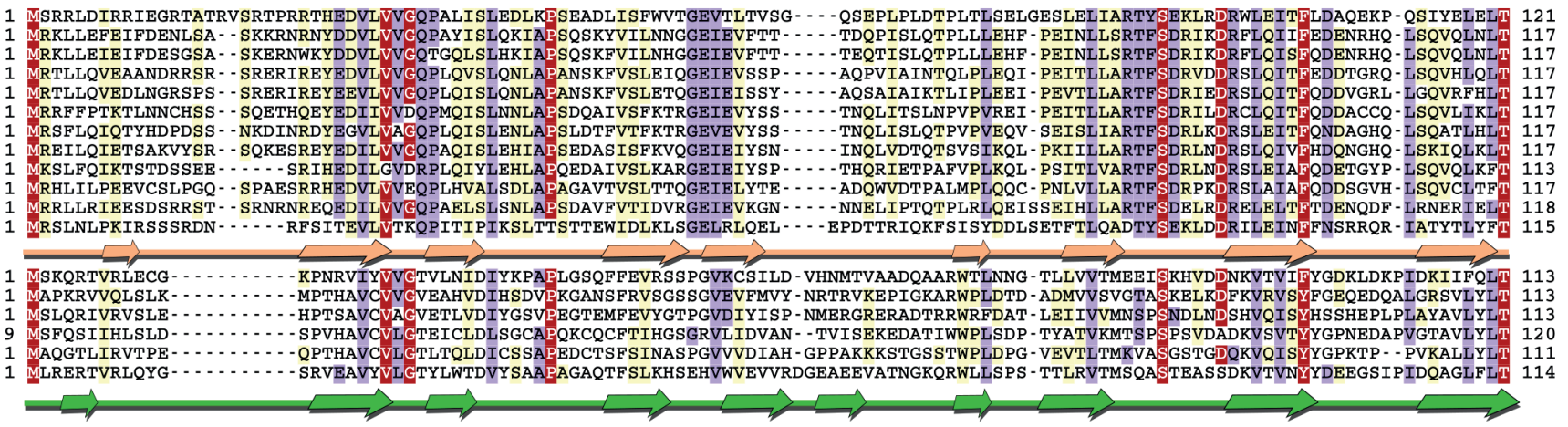

b made available under aCC-BY-NC-ND 4.0 International license.

Beta strand connecting PAD_M and C domains 290

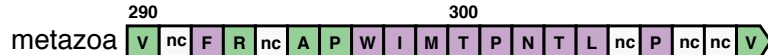

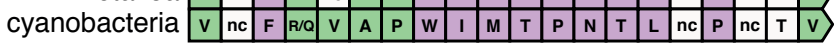

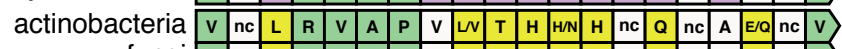
\begin{tabular}{llllllllllll|l|l|l|l|l|l|l|l|l|l|l|} 
fungi & V & nc & L & R & V & A & P & I & L & T & H & H & H & nc & Q & nc & V & Q & Q & V \\
\cline { 2 - 6 }
\end{tabular}

Calcium Calcium Calcium $\quad$ Calcium binding site 6 binding site 3 binding site 4 binding site 5

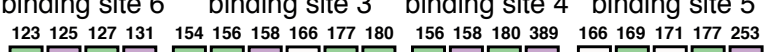

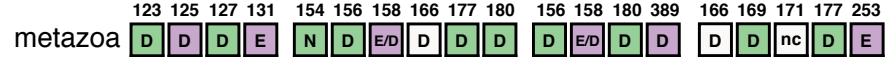

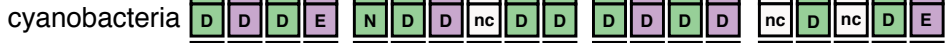

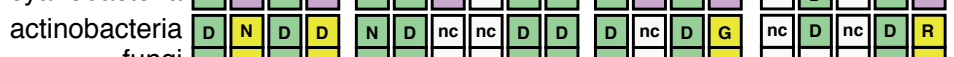

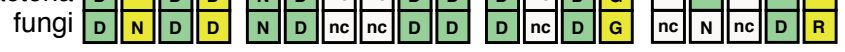

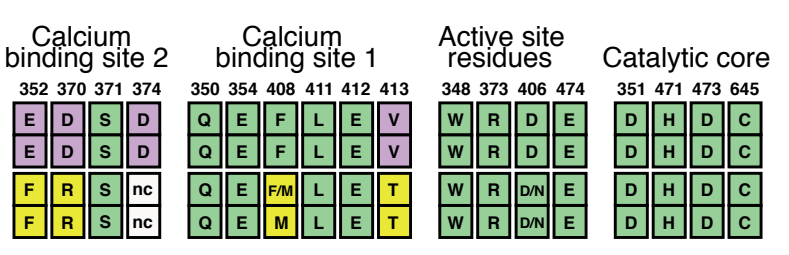

Region within Ca3-5 binding site

\begin{tabular}{|c|c|c|c|c|c|c|c|c|c|c|c|c|c|c|c|c|c|c|c|c|}
\hline & & & & & & & & & & & & & & & & & & & & 180 \\
\hline $\mathbf{N}$ & & & [ & nc & & $\mathrm{n}$ & $n c$ & nc & U & $\mathrm{nc}$ & $\mathrm{nc}$ & $n c$ & $\mathrm{nc}$ & $G / S$ & $n c$ & $\mathrm{nc}$ & $\bar{D}$ & $\mathrm{~L}$ & KL & $\mathrm{D}$ \\
\hline $\mathbf{N}$ & 5 & & D & $\mathrm{nc}$ & D & $\mathrm{nc}$ & $\mathrm{nc}$ & $n c$ & $D$ & $n c$ & $\mathrm{nc}$ & $\mathrm{nc}$ & $\mathrm{nc}$ & $\bar{G}$ & $\mathrm{~L}$ & L & $D$ & $\mathrm{LI}$ & $\mathrm{K}$ & $D$ \\
\hline $\bar{N}$ & I & G & D & $\overline{\mathrm{nc}}$ & $\overline{\mathrm{nc}}$ & $\mathrm{K} / \mathrm{R}$ & $\mathbf{R}$ & C & D & $\overline{\mathrm{nc}}$ & $\mathrm{nc}$ & $\overline{L L}$ & $\mathrm{~A} / \mathrm{E}$ & $\bar{A}$ & c & $\mathbf{N}$ & $\bar{D}$ & A & $s / \mathrm{Q}$ & $\bar{D}$ \\
\hline $\mathbf{N}$ & 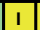 & $G$ & & $\bar{T}$ & nc & $P$ & $\mathbf{R}$ & & N & $E$ & $\mathrm{nc}$ & L & A & $\overrightarrow{\mathbf{A}}$ & $c$ & $\mathbf{N}$ & D & $\mathbf{A}$ & $\mathrm{s}$ & D \\
\hline
\end{tabular}

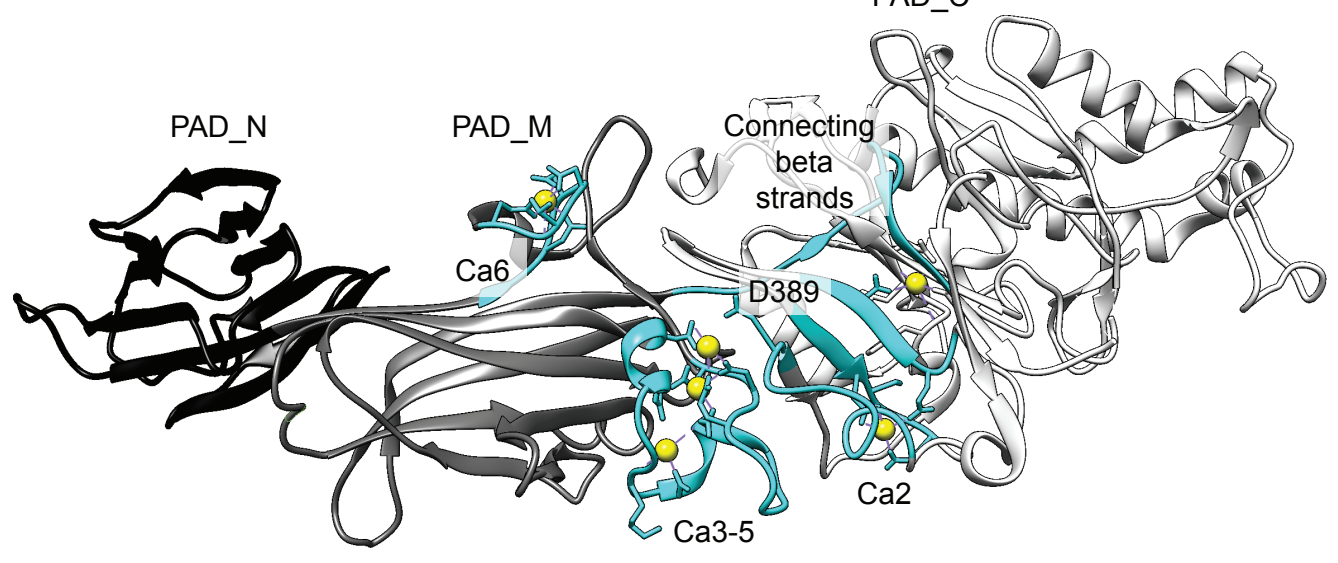

Figure 2: Synapomorphic features among PADI orthologues. a) Alignment of putative PAD_N domains from SPM/NX clade cyanobacterial PADI sequences with the PAD_N domain from human PADI paralogues and Rhincodon typus (whale shark). The colouring scheme indicates the average BLOSUM62 scores of each alignment column: red (>3.5), violet (between 3.5 and 2) and light yellow (between 2 and 0.5). Peach arrows shown below the cyanobacterial sequences indicate PsiPred predicted secondary structure (beta sheets). Green arrows (beta sheets) correspond to the known secondary structure of the PAD_N domain of human PADI2. b) Analysis of synapomorphic regions, representing six PADI sequences from each of metazoa, cyanobacteria, actinobacteria and fungi. Consensus sites across the six species are shown with standard single letter amino acid abbreviations. "nc" (non-nonserved) represents the absence of consensus conservation to one or two amino acids across the six species. The numbering given above the alignment and corresponds to the ungapped site of human PADI2 such that residues can be compared to Slade et al. Sites showing conservation across all four domains are coloured in green, sites showing synapomorphy between metazoa and cyanobacteria are coloured in purple, and sites showing synapomorphy between fungi and actinobacteria are coloured in yellow. c) Crystal structure of human PADI2 presented with PAD_N domain coloured in black, PAD_M domain in grey and PAD_C domain in white. Synapomorphic regions are coloured in cyan and calcium ions are shown as yellow spheres. 
bioRxiv preprint doi: https://doi.org/10.1101/2020.06.13.150037; this version posted June 15, 2020. The copyright holder for this preprint Figure(1) (Bich was not certified by peer review) is the author/funder, who has granted bioRxiv a license to display the preprint in perpetuity. It is
made available under aCC-BY-NC-ND 4.0 International license.

\section{a}

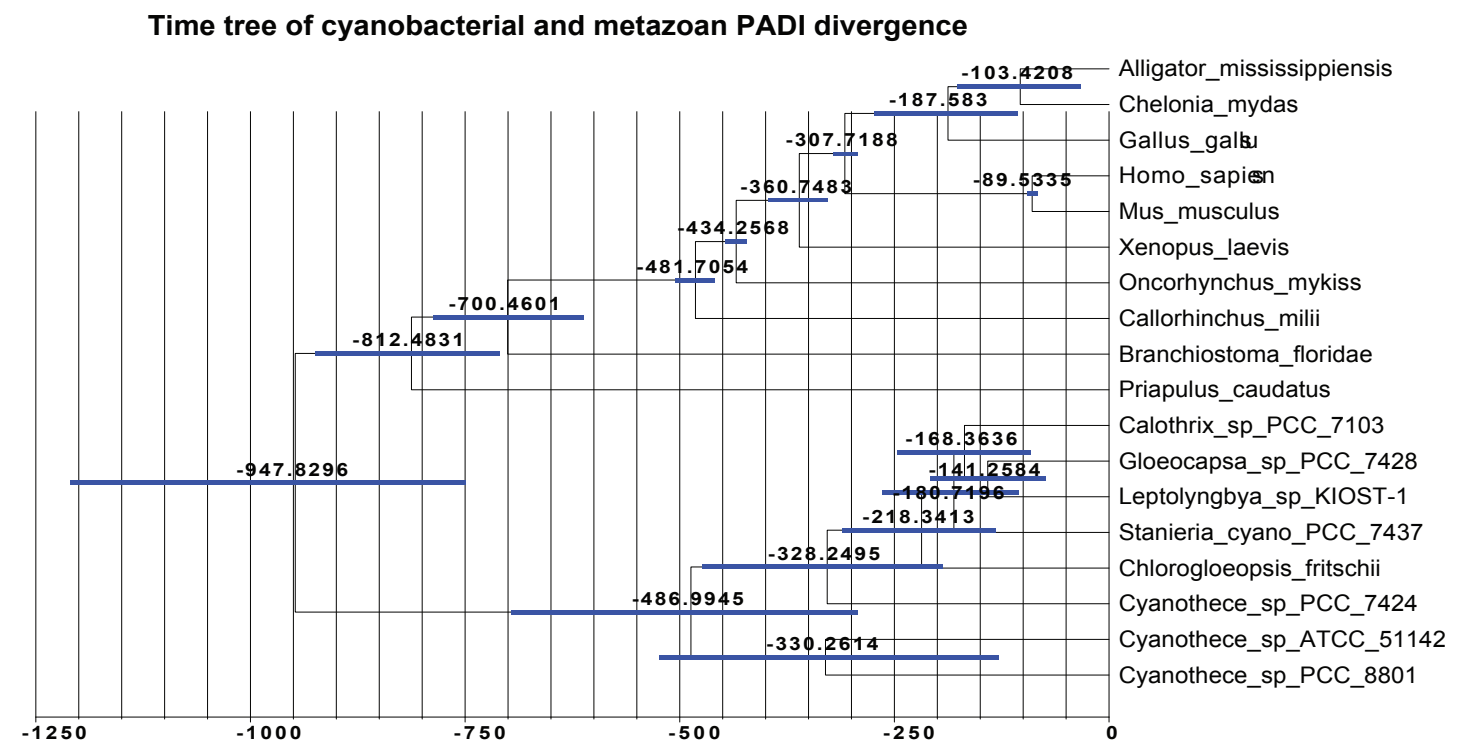

b

Estimated divergence time of cyanobacterial and metazoan PADls

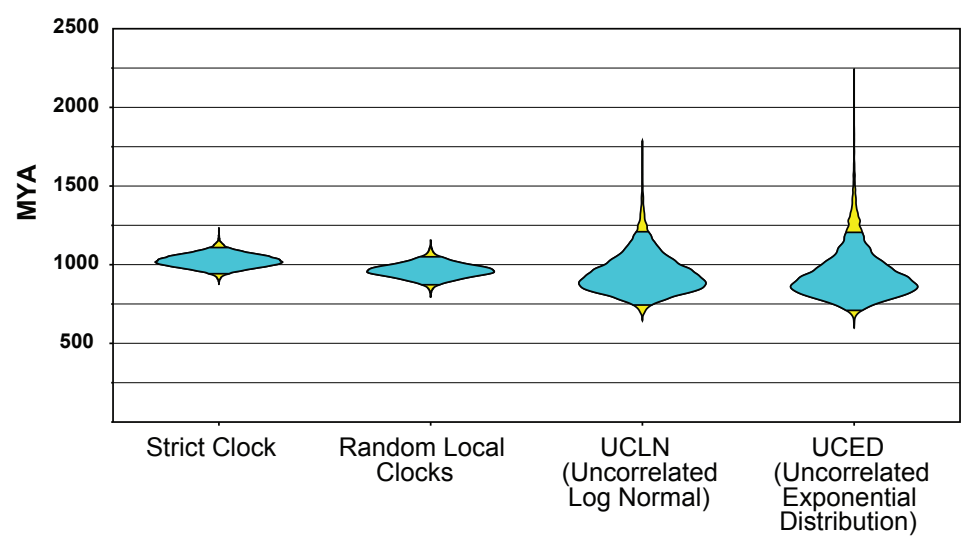

C

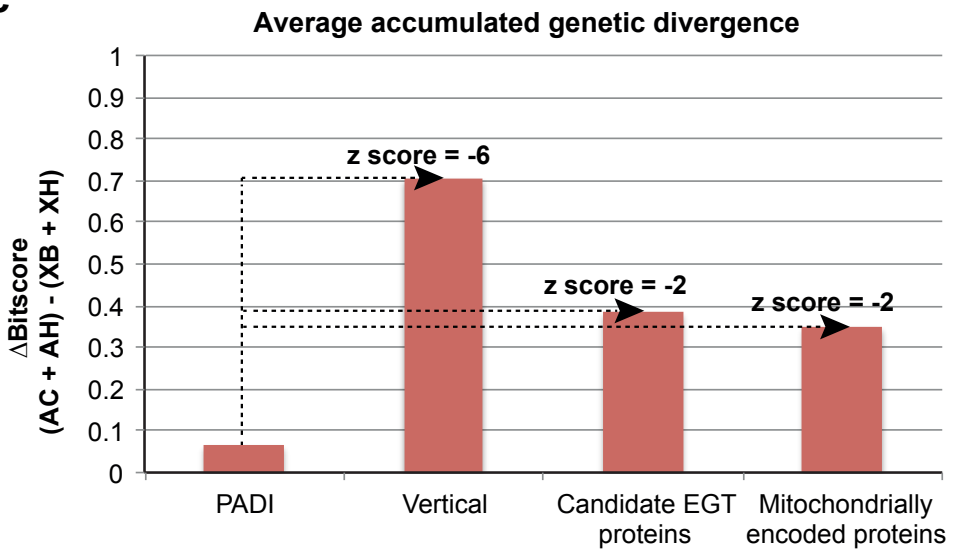

Figure 3: PADI sequence divergence analyses. a,b) Estimated divergence time of cyanobacteria and metazoa based on PADI sequences with respect to geologically defined constraints from the fossil record. a) Metazoan sequences and SPM/NX clade cyanobacterial sequences taken from the PATRIC database were used for Bayesian phylogenetic analysis in BEAST under the uncorrelated lognormal (UCLN) clock model, using a calibrated Yule model as the tree prior. Divergence times from the fossil record are used as normally distributed priors for six different nodes from metazoa. The marginal posterior distribution of the age of the root of the whole tree is used to estimate the divergence time; all nodes are labelled with the 95\% credible interval for the marginal posterior distributions of the node ages. b) Estimated divergence times for running the analysis under different clock models (strict clock, random local clocks, UCLN and UCED), with the marginal posterior distribution of the age of the root of the whole tree given as a violin plot, where the $95 \%$ credible interval is given in cyan and the limits coloured in yellow. c) Analysis of the Accumulated Genetic Divergence (AGD) of PADIs compared to highly conserved proteins. The AGD was calculated for 26 vertically transferred proteins, 19 candidate EGT proteins, and 10 proteins encoded in the mitochondrial genome. The mean AGD for those groups is plotted against the AGD for PADI proteins. 
a

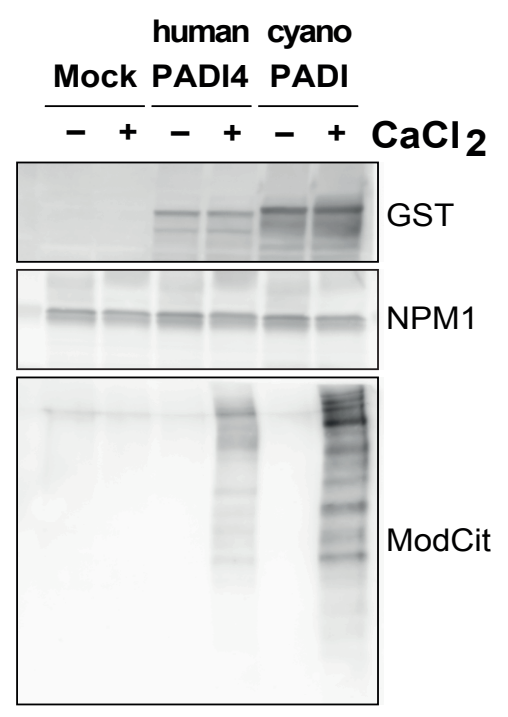

b

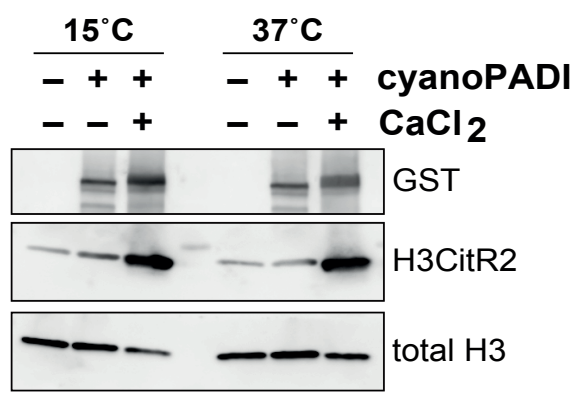

Figure 4: Biochemical analyses of the cyanobacterial PADI enzyme from Cyanothece sp. 8801 (cyanoPADI). Immunoblot analyses of citrullination assays using GST-His-tagged recombinant enzymes. a) Whole cell lysates from mouse embryonic stem cells were used as substrate and the presence of citrullination in a protein sequenceindependent manner was assessed using the ModCit antibody. Nucleophosmin (NPM1) is used as a loading control. b) Recombinant human histone $\mathrm{H} 3$ was used as substrate and citrullination of arginine 2 was assessed. Total histone $\mathrm{H} 3$ is used as loading control. 
a

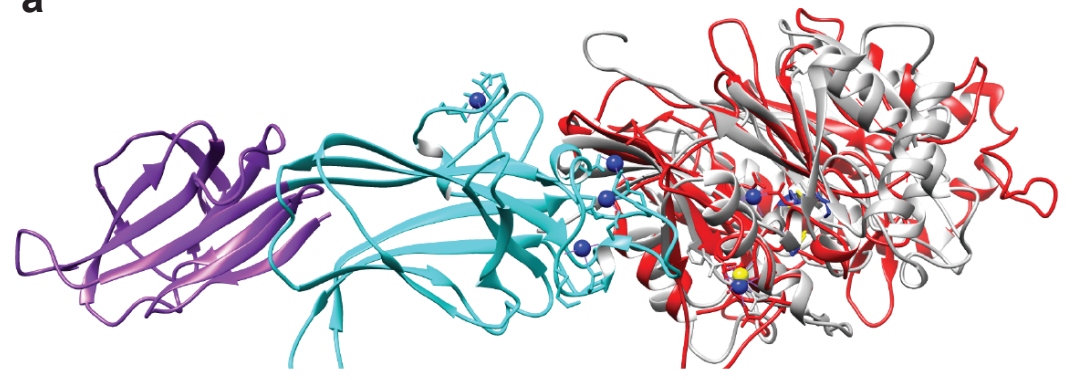

b

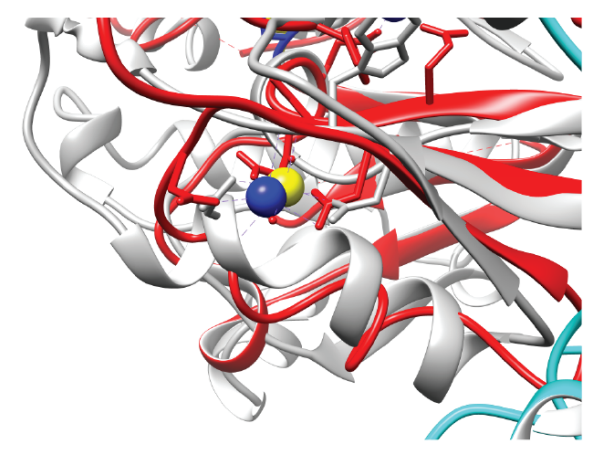

C

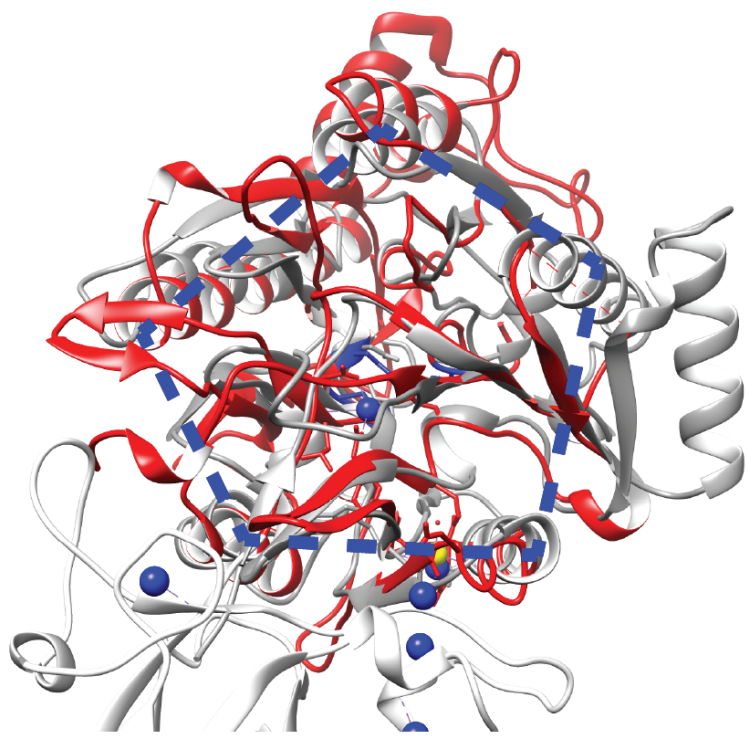

d

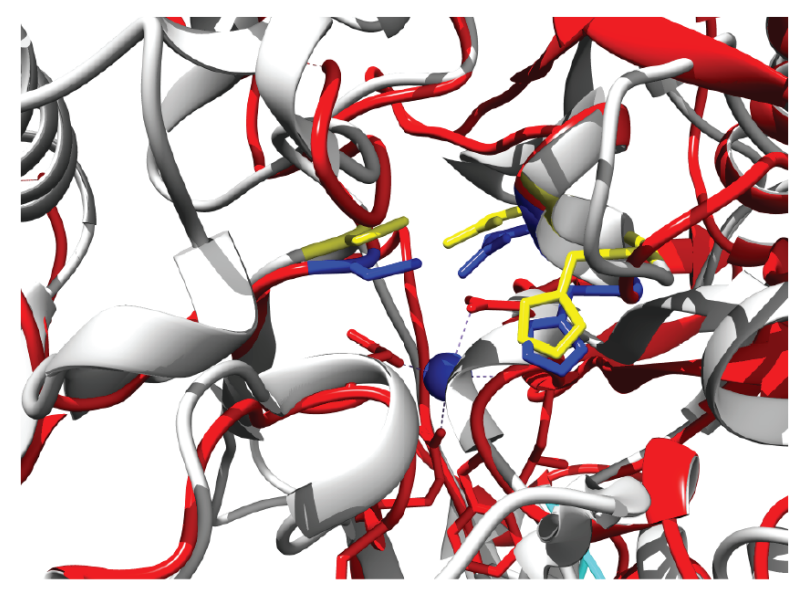

Figure S1: The active site of human PADI2 adopts an ancient configuration. PADI2 structure (PDB: 4n2c) in purple (PAD_N domain), cyan (PAD_M domain) and red (PAD_C domain) was superimposed with the structure of Agmatine deiminase from $\bar{C}$ hlorobium tepidum (ctAgD) in grey (PDB: $1 \times \mathrm{kn}$ ). a) Full structure of PADI2 shows that Agmatine deminase (grey) superimposes onto the PAD_C pfam domain (red). b) Detail of the conserved metal ion binding site between the two enzymes (Calcium binding site 2 in PADI2, blue sphere, and $\mathrm{Na}+$ site in ctAgD, yellow sphere). c) Detail of the PAD_C domain shows the conservation of the overall pentein fold (blue dotted line shows five-fold rotational symmetry). d) Active site configuration and positioning is conserved between PADI2 (blue: His-Asp-Cys triad) and ctAgD (yellow: His-Asp-Cys triad). 


\section{Figure S2}

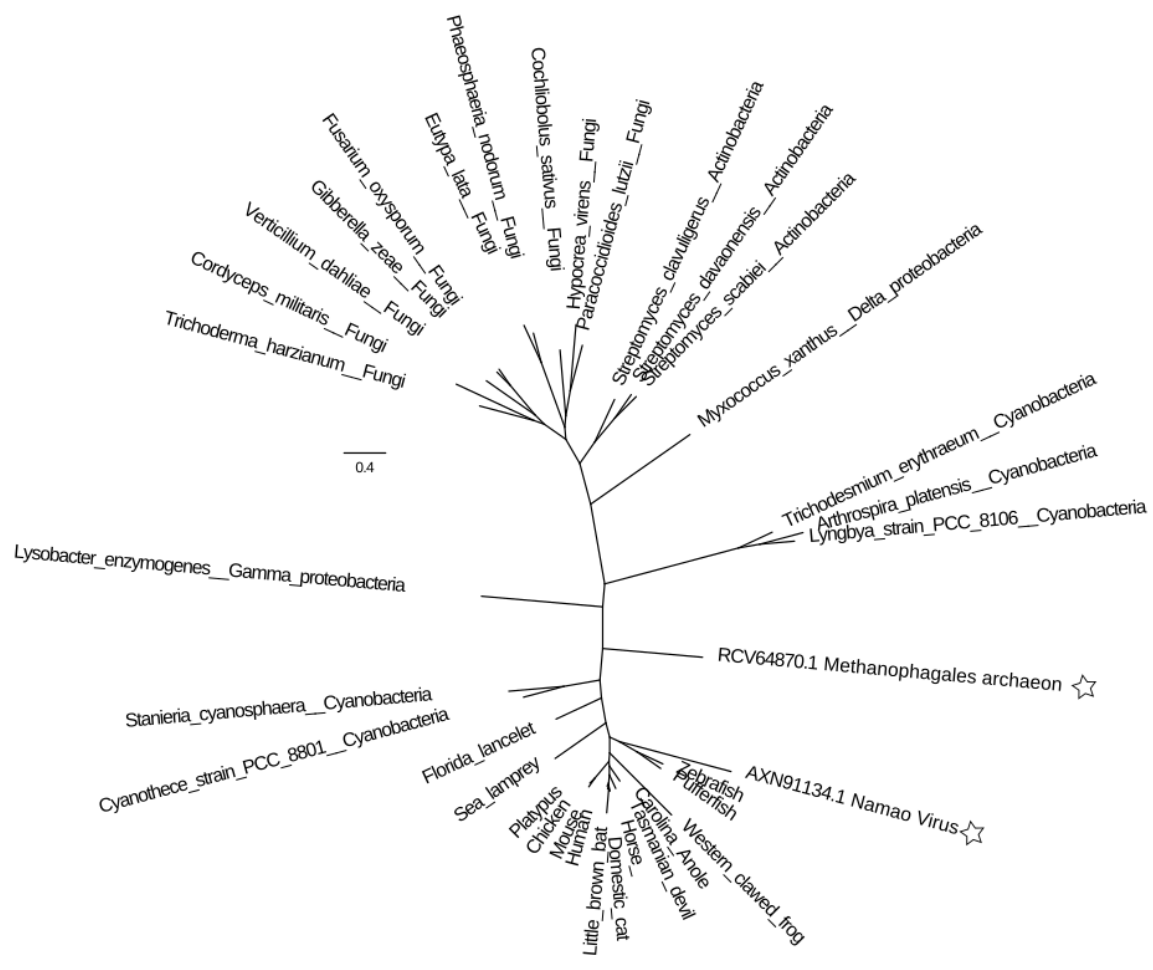

Figure S2: Phylogeny of two spurious putative PADI sequences from unexpected clades within the core set of $P A D I$ sequences. The putative $P A D I$ homologues from Namao virus and Methanophagales archeon are indicated with a star icon. The phylogeny of PADI homologues places the Namao virus sequence within the clade containing bony fish. Since this virus is known to infect Acipenser fulvescens (lake sturgeon) and the particular genome in question was isolated from an infected tissue sample, it is highly likely that this sequence is the result of sample contamination from the host organism. The Methanophagales archeon sequence is placed very close to bacterial sequences at a distance which is inconsistent with even the slow divergence of core housekeeping genes shared between bacteria and archaea. As $P A D I$ does not appear to be part of the core archaeal genome as it is not a housekeeping gene vital for archaeal cell survival, it is unlikely that this sequence would show such a slow rate of evolution while also being lost simultaneously within all other archaeal species. 
bioRxiv preprint doi: https://doi org/10.1101/2020 06 13 150037 this version posted June 15,2020 . The copyriaht holder for this preprint Figurewhighas not certified by peer review) is the author/funder, who has granted bioRxiv a license to display the preprint in perpetuity. It is

a made available under aCC-BY-NC-ND 4.0 International license.

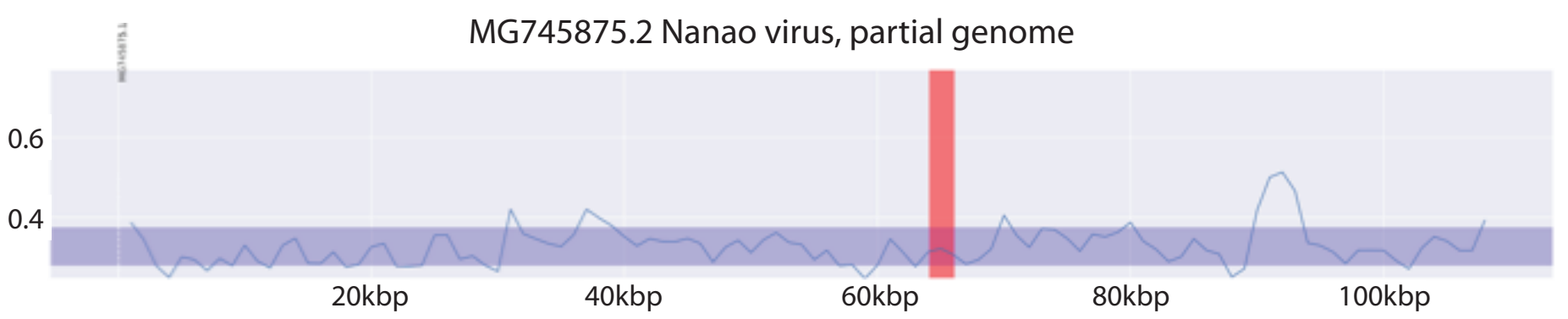

b

QENH01000201.1 Methanophagales archaeon, isolate whole genome shotgun sequence

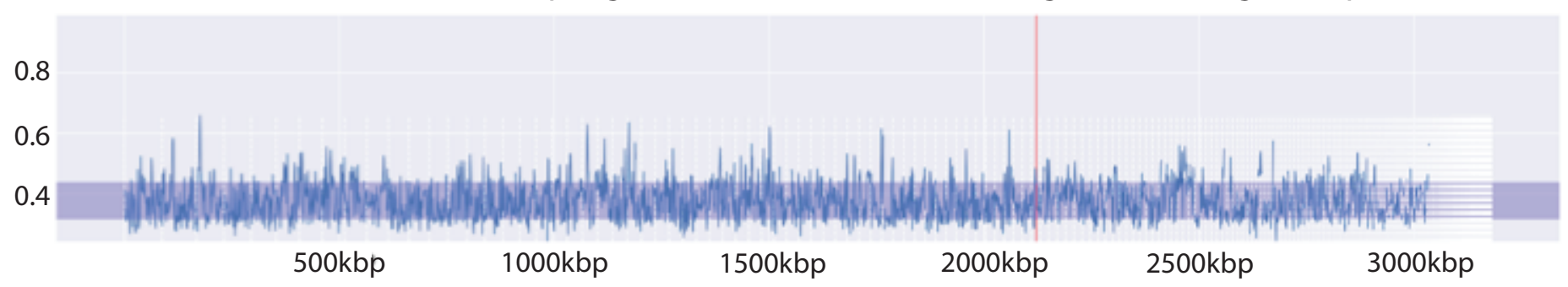

Figure S3: K-mer spectra analysis of genomic contigs of spurious viral (a) and archaeal (b) hits.

The norm of the vector defined by the difference between the genomic average and the K-mer spectrum of each window is shown over all contigs in both assemblies. Vertical white dashed lines denote the start of a contig. The horizontal blue shaded region shows the standard deviation around the average distance of all windows relative to the genomic average. The red vertical shaded area shows the position of the putative PADI homologue within the genome. Contig identifiers are not shown for the genome of Methanophagales archeon due to the high number of small contigs. The assembly quality of this genome is fairly poor, contigs are ordered by length and do not represent the actual arrangement. K-mer spectra for contigs shorter than $1 \mathrm{kbp}$ were not calculated. Neither of the genomes present a region showing anomalous $\mathrm{K}$-mer spectra typical of a continuous horizontally transferred genomic region. This indicates that the two PADI sequences were falsely attributed to these genomes during assembly due to their $\mathrm{K}$-mer spectra being close to the genomic average of these two organisms by chance. 

Figure $S 4$ made available under aCC-BY-NC-ND 4.0 International license.

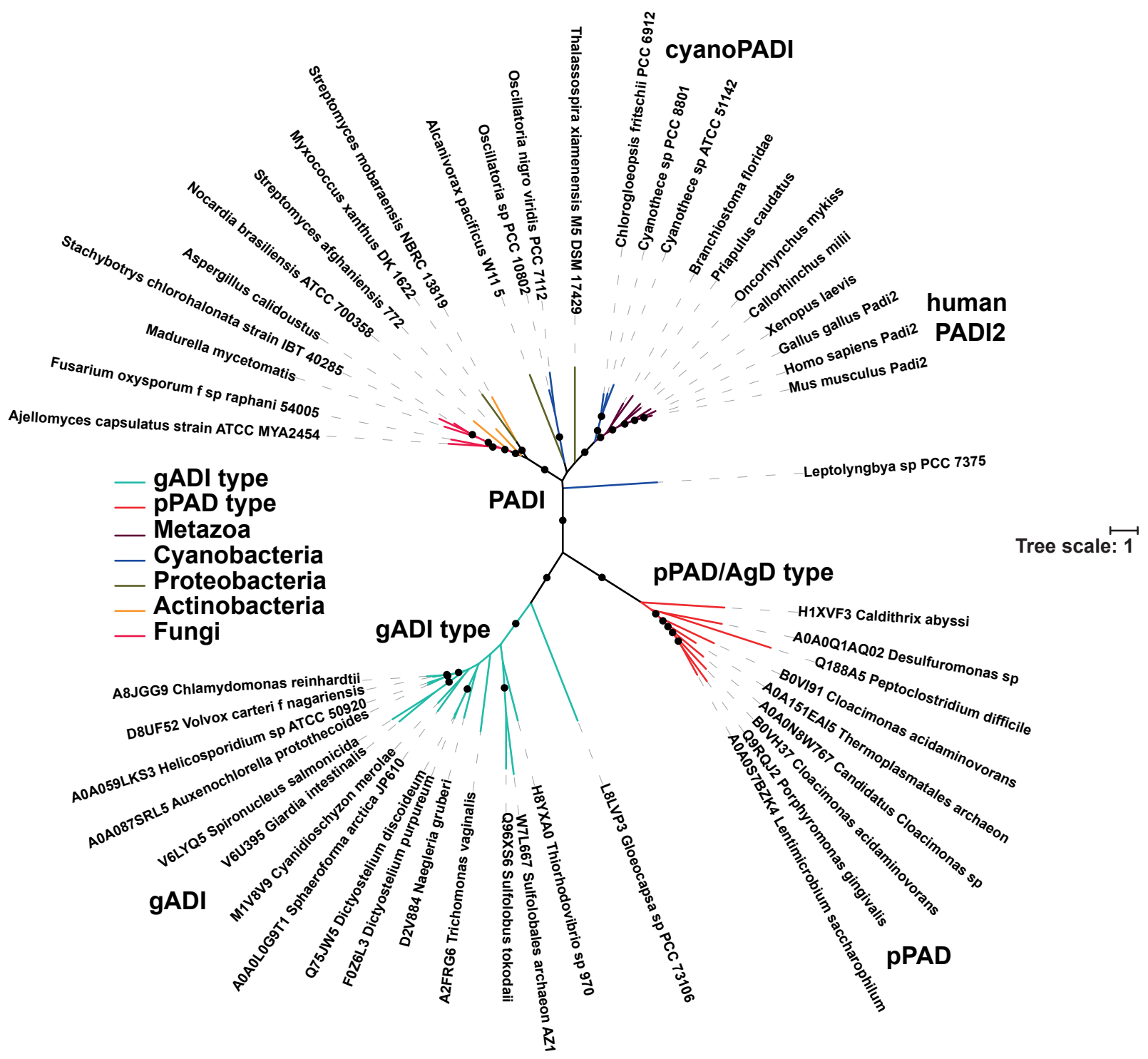

Figure S4: Metazoan PADI sequences are evolutionarily distinct from other bacterial and eukaryotic citrullinating enzymes. PADls form distinct clades to ADls, AgDs, gADI (arginine deiminase from Giardia lamblia) and PPAD (porphyromonas-type peptidylarginine deiminase from Porphyromonas gingivalis) sequences. The tree is shown unrooted with solid circles indicating consensus node support of $>95 \%$. 
bioRxiv preprint doi: https://doi.org/10.1101/2020.06.13.150037; this version posted June 15, 2020. The copyright holder for this preprint (which was not certified by peer review) is the author/funder, who has granted bioRxiv a license to display the preprint in perpetuity. It is

Figure S5 made available under aCC-BY-NC-ND 4.0 International license.

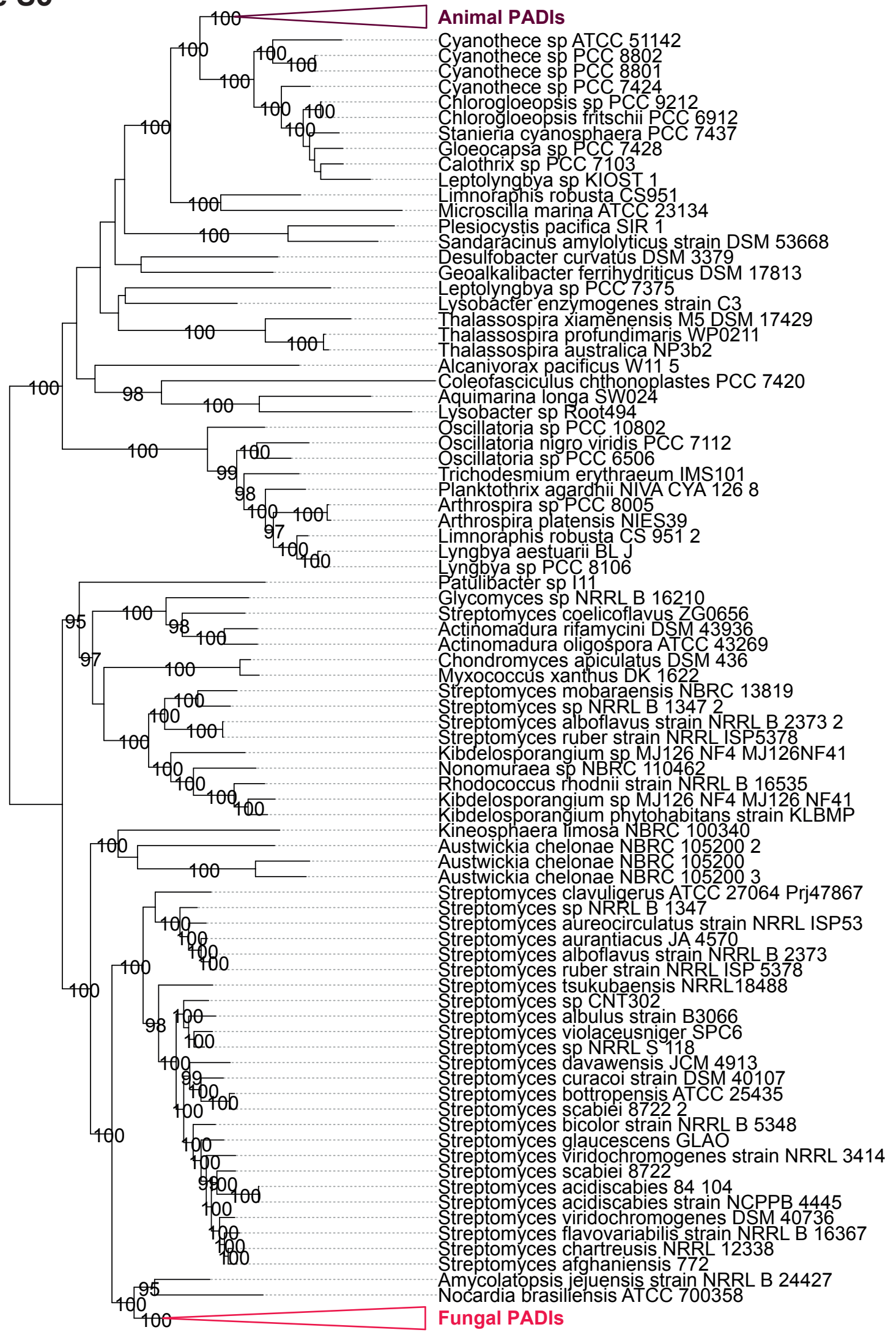

Figure S5: PADI phylogeny. Phylogenetic analysis of all putative bacterial PADI sequences in the PATRIC database and with representative animal and fungal sequences. The tree is shown rooted at the midpoint with solid circles indicating consensus node support of $>95 \%$ and a number of critical nodes labeled in full. 
bioRxiv preprint doi: https://doi.org/10.1101/2020.06.13.150037; this version posted June 15, 2020. The copyright holder for this preprint (which was not certified by peer review) is the author/funder, who has granted bioRxiv a license to display the preprint in perpetuity. It is Figure S6 made available under aCC-BY-NC-ND 4.0 International license.

a

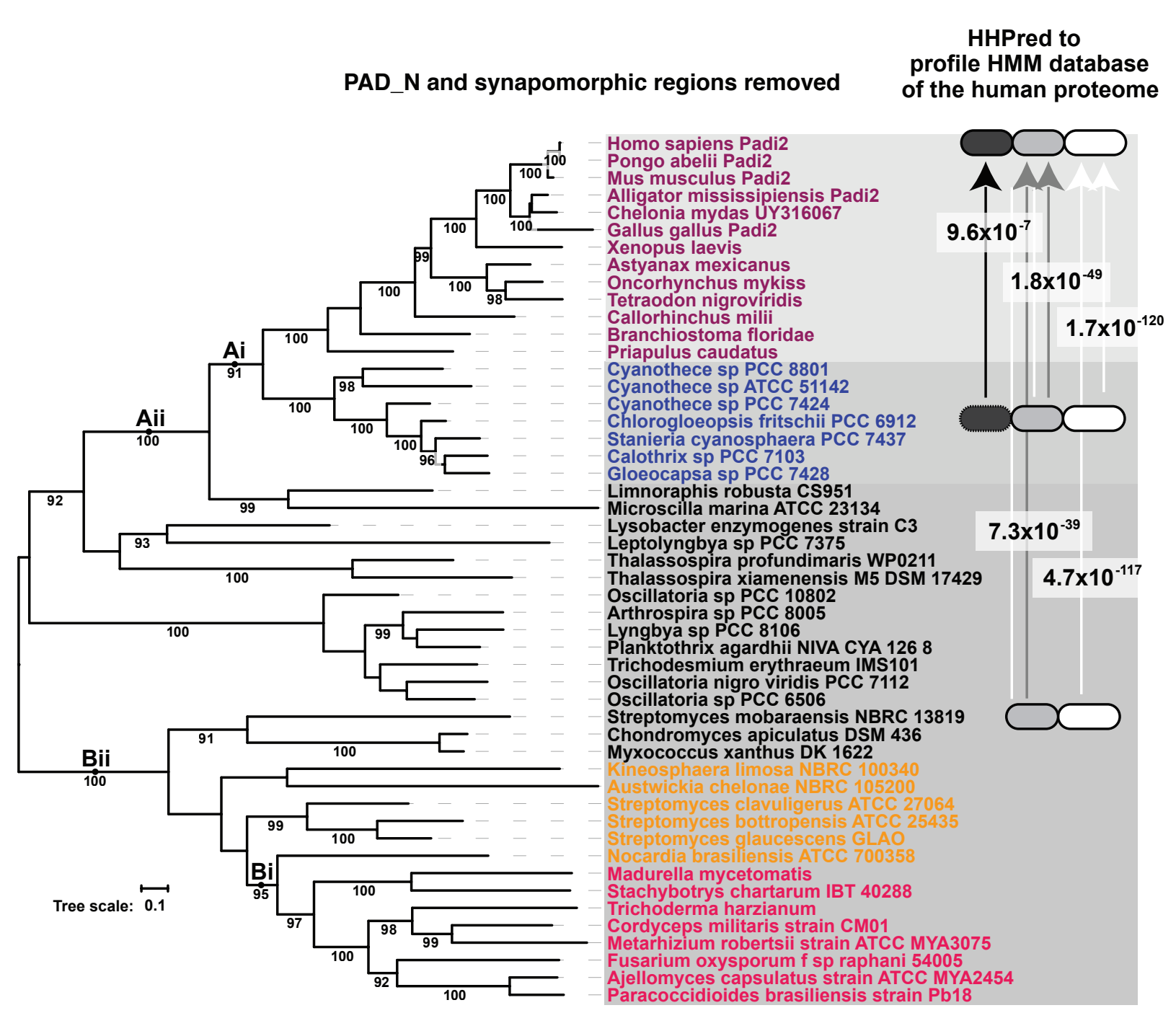

b

\begin{tabular}{|c|c|c|c|c|c|}
\hline \multirow{2}{*}{ Cartoon: } & \multirow{2}{*}{ Region of alignment used: } & \multicolumn{4}{|c|}{ Node support for clade: } \\
\hline & & $\mathbf{A i}$ & Aii & $\mathrm{Bi}$ & Bii \\
\hline & Whole protein & 99 & 100 & 99 & 100 \\
\hline & PAD_N removed & 97 & 100 & 99 & 100 \\
\hline$\star$ & $\begin{array}{c}\text { PAD_N and } \\
\text { synapomorphy removed }\end{array}$ & 91 & 100 & 95 & 100 \\
\hline
\end{tabular}

Figure S6: Domain architecture analysis of PADI orthologues. a) Representation of PADI domain architecture across the phylogenetic tree. Protein regions aligning to the metazoan PADI domains were extracted from sequences represented in the different clades of the tree (firstly of cyanobacterial sequences and secondly of a mixture of bacterial and fungal sequences). An HMM profile was made and HHPred was used to search against a database of profiles made of the entire human proteome, and against a database of Pfam domain profiles. The E-values are given for these searches where significant sequence similarity could be identified from HHPred searches. b) Phylogenetic analyses from Figure 1a (top row) were repeated using an alignment where the PAD_N domain (middle row), or both the PAD_N domain and regions of synapomorphy (bottom row) were removed. Maximum likelihood inference using IQTree was used in all three cases and ModelFinder was used to select the best performing fixed empirical rate matrix (WAG + R5 +F0). Node support values correspond to clades annotated in Figure 1a as topologies were congruent. 


\section{Vertical trajectory:}

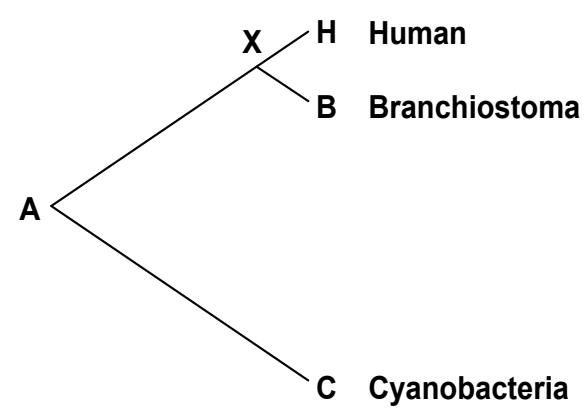

Accumulated genetic divergence $=$ $\Delta$ Bitscore (Cy-Hu) - $\Delta$ Bitscore(Br-Hu)

$(A C+A H)-(X B+X H)$

$=(A C+A X)-X B$

If vertical, expect $(A C+A X)-X B>>0$

\section{Horizontal trajectory:}

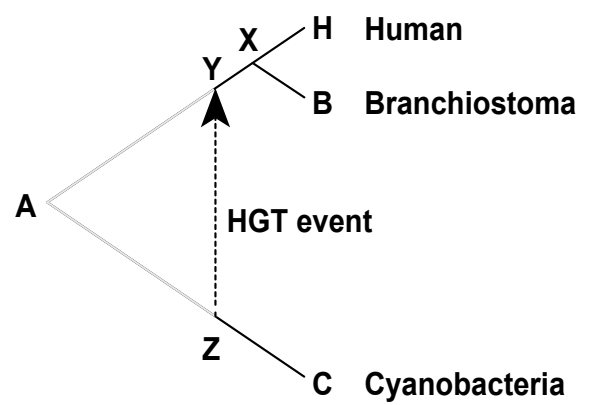

Accumulated genetic divergence $=$ $\Delta$ Bitscore $(\mathrm{Cy}-\mathrm{Hu})-\Delta \mathrm{Bitscore}(\mathrm{Br}-\mathrm{Hu})$ $(A C+A H)-(X B+X H)$

If horizontal, $\mathrm{AC}=\mathrm{ZC}$ and $\mathrm{AH}=\mathrm{YH}$

$\Rightarrow(\mathrm{ZC}+\mathrm{YH})-(\mathrm{XB}+\mathrm{XH})$

$=(Z C+Y X)-X B$

If $Y X \approx 0$, then $(Z C+Y X)-X B$ can be $\approx 0$

Figure S7: Calculating the AGD of a given protein between its homologues in Homo sapiens, Branchiostoma spp. and Cyanothece spp. The bitscore density of the similarity of the cyanobacterial homologue to the human sequence $\Delta$ bitscore $_{\mathrm{Cy}-\mathrm{Hu}}(\mathrm{AC}+\mathrm{AH})$ and the bitscore density of the similarity of the branchiostomal homologue to the human sequence $\Delta$ bitscore $_{\mathrm{Br}-\mathrm{Hu}}(\mathrm{XB}+\mathrm{XH})$ are calculated. In a vertical scenario the $A G D$, given by $(A C+A X)-X B$, will be much greater than zero. In a horizontal scenario, $(Z C+Y X)$ may be approximately equal to $X B$ and so the $A G D$, given by $(Z C+Y X)-X B$ may be close to zero. 

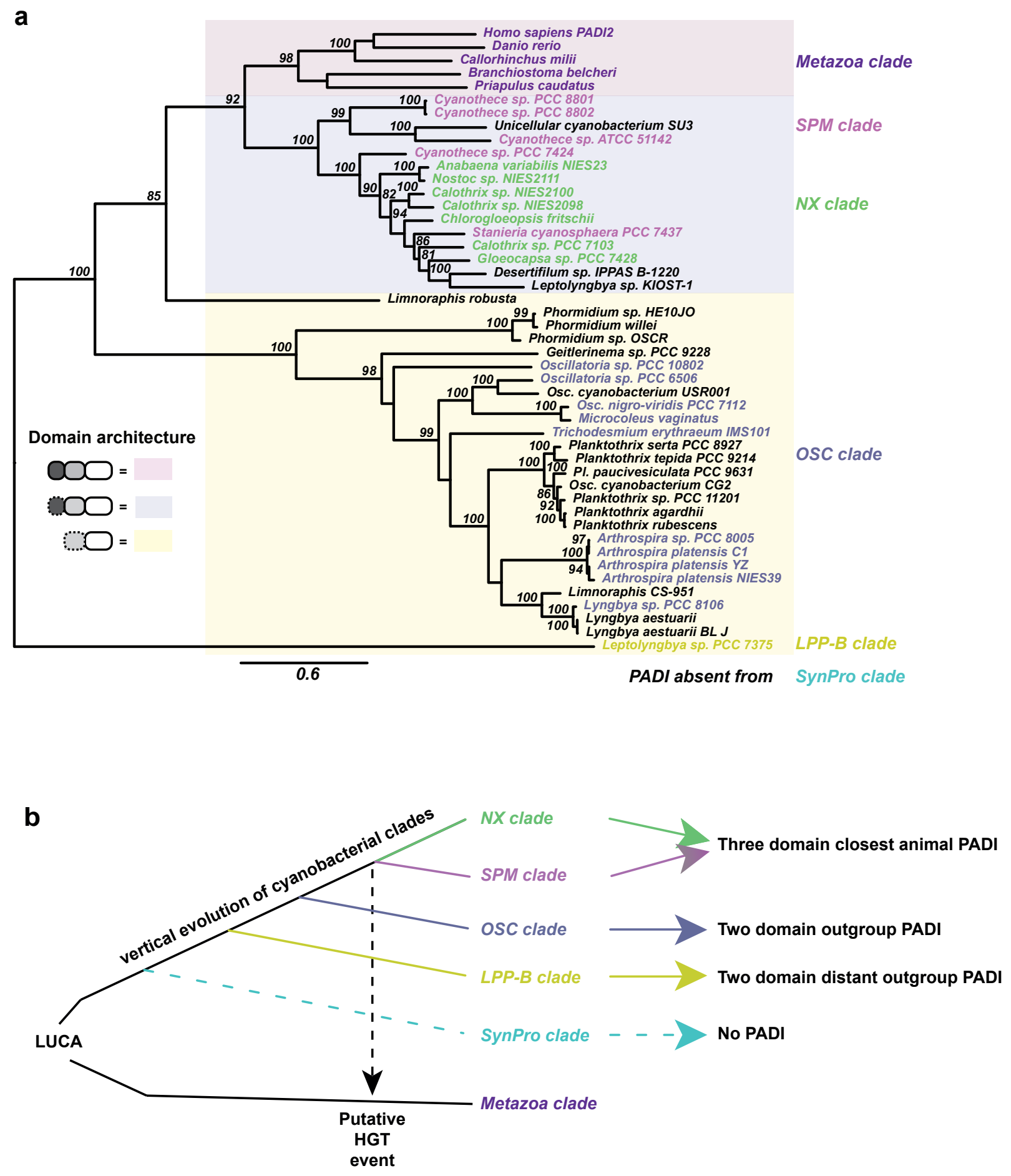

Figure S8: Cyanobacterial origin of the PADI sequence. a) Phylogenetic analysis of cyanobacterial PADI sequences reproduces the known clades of cyanobacterial evolution. Colours and names of clades are used as in Uyeda et al. Species that were not analysed in Uyeda et al. are in black. Sequences were aligned with MUSCLE and the tree was built using PhyML 3.0. Bootstrap support $>80 \%$ is indicated on branches from 250 bootstrap replicates. The domain architecture of each sequence was analysed and is represented in the legend. b) Schematic showing the proposed HGT event from cyanobacteria to metazoa. PADI present in a last common ancestor of the NX/SPM clades of cyanobacteria, which possess a three-domain PADI, and was transferred to an ancient last common ancestor in the metazoan lineage. 
bioRxiv preprint doi: https://doi.org/10.1101/2020.06.13.150037: this version posted June 15, 2020. The copyright holder for this preprint (which was not certified by peer review) is the author/funder, who has granted bioRxiv a license to display the preprint in perpetuity. It is Figure $\mathbf{S 9}$ made available under aCC-BY-NC-ND 4.0 International license.

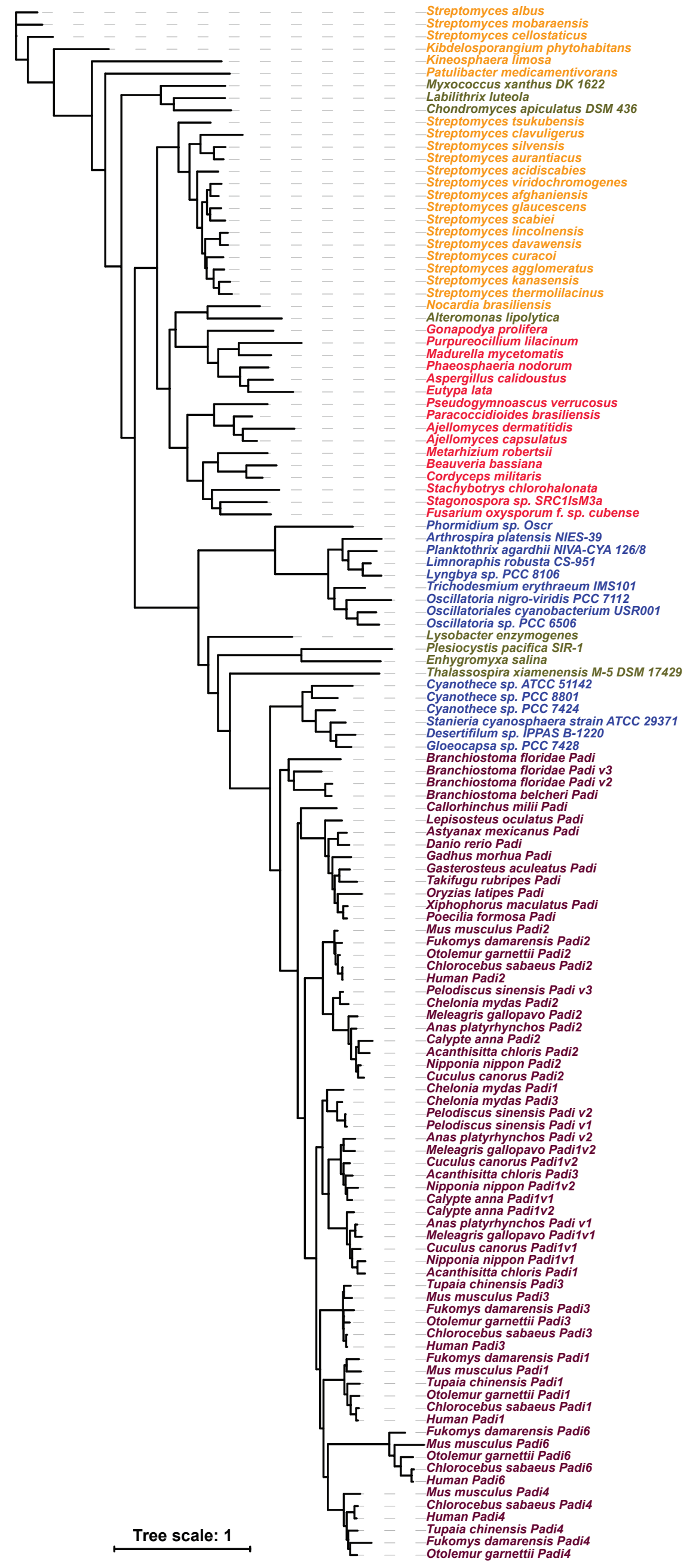

Actinobacterial Padi

Proteobacterial Padi

Actinobacterial Padi

Proteobacterial Padi

Fungal Padi

Cyanobacterial Padi

Proteobacterial Padi

SPM/NX Padi

Branchiostoma Padi

Fish Padi

Padi2

PADI duplications

Padi v1

Padi v2

Padi3

Padi1

Padi6

Padi4 
bioRxiv preprint doi: https://doi.org/10.1101/2020.06.13.150037; this version posted June 15, 2020. The copyright holder for this preprint (which was not certified by peer review) is the author/funder, who has granted bioRxiv a license to display the preprint in perpetuity. It is made available under aCC-BY-NC-ND 4.0 International license.

Figure S9: The PADI sequence was retained and underwent multiple duplications in metazoa. Phylogenetic analysis of a large number of putative PADI sequences showing the multiple duplications in metazoa. The first duplication produced two new orthologues present in reptiles and birds which cluster in their own groups denoted PADIv1 and PADlv2. Subsequent duplications in mammals produced the separation of clusters of PADI1, PADI3, PADI4 and PADI6. 